\title{
NUMERICAL ANALYSIS OF A FINITE ELEMENT METHOD TO COMPUTE THE VIBRATION MODES OF A REISSNER-MINDLIN LAMINATED PLATE
}

\author{
RICARDO G. DURÁN, RODOLFO RODRÍGUEZ, AND FRANK SANHUEZA
}

\begin{abstract}
This paper deals with the finite element approximation of the vibration modes of a laminated plate modeled by the Reissner-Mindlin equations; DL3 elements are used for the bending terms and standard piecewise linear continuous elements for the in-plane displacements. An a priori estimate of the regularity of the solution, independent of the plate thickness, is proved for the corresponding load problem. This allows using the abstract approximation theory for spectral problems to study the convergence of the proposed finite element method. Thus, optimal order error estimates including a double order for the vibration frequencies are obtained under appropriate assumptions. These estimates are independent of the plate thickness, which leads to the conclusion that the method is locking-free. Numerical tests are reported to assess the performance of the method.
\end{abstract}

\section{INTRODUCTION}

The laminated plates are widely used in engineering practice, for instance, in automobile, space, and civil applications. The main motivation for this interest is related to the improved ratio between performances and weight of this kind of plates with respect to homogeneous ones. Several different models of these plates have been proposed. The simplest one is the Classical Laminated Plate Theory (CLPT) [13, which is based on the Kirchhoff hypotheses. However, other models arising from the Reissner-Mindlin assumptions are often preferred; they are called First-order Shear-Deformation Theory (FSDT) 3].

Locking is a very well-known phenomenon in the numerical computation of plate problems. It consists of the fact that very unsatisfactory results are obtained when the thickness is small with respect to the other dimensions of the structure. From the point of view of the numerical analysis, locking reveals itself in that the $a$ priori estimates depend on the thickness of the structure in such a way that they degenerate when this parameter becomes small. Several methods based on reduced

Received by the editor August 24, 2009 and, in revised form, June 9, 2010.

2010 Mathematics Subject Classification. Primary 65N25, 74K10, 65N30.

Key words and phrases. Reissner-Mindlin, laminated plates, spectral problems.

The first author was partially supported by Universidad de Buenos Aires under grant X070. Member of CONICET (Argentina).

The second author was partially supported by FONDAP and BASAL projects CMM, Universidad de Chile (Chile).

The third author was supported by a CONICYT fellowship (Chile).

All authors were partially supported by ANPCyT through grant PICT RAÍCES 2006, No. 1307 (Argentina).

(c)2011 American Mathematical Society 1239

Reverts to public domain 28 years from publication 
integration or mixed formulations have been devised to avoid locking in the load plate problem (see [6], for instance). Some of them have been analyzed for the plate vibration problem, as well. For instance, the DL3 and the MITC elements (which were introduced for load problems in [10] and [5], respectively) have been analyzed for vibration problems in [9] and [8, respectively.

Locking-free methods for load problems of laminated plates with several layers all of the same thickness have been analyzed in 2] in a general context. In the present paper we address the corresponding vibration problem. For simplicity, we present the analysis in the case of only two layers. We use the DL3 elements introduced in [10] for the bending terms and standard triangular finite elements for the in-plane displacements. The analysis is made in the framework of the abstract spectral approximation theory for compact operators as stated, for instance, in [4, Section 7]. The goal is to prove optimal order convergence in $L^{2}$ and $H^{1}$ norms for the eigenfunctions and a double order for the eigenvalues. Moreover, to ensure that the method is locking-free, it has to be proved that the error estimates do not deteriorate as the plate thickness becomes small.

The present analysis is based on the results for load problems for laminated plates from [2] and for the vibration problem for homogeneous plates from [9]. The main difficulty is the need for additional regularity of the solution of the load problem for a Reissner-Mindlin laminated plate. This is the reason why we had to prove new a priori estimates for this problem, with constants independent of the plate thickness. This result is interesting by itself, since the error of any numerical method for laminated plates will rely on such an estimate. To the best of the authors' knowledge, the obtained estimate had not been proved before. Moreover, the proof of the analogous estimate known for classical Reissner-Mindlin plates (see 1]) does not extend directly to this case. In fact, to extend it, we had to resort to more recent regularity results for formally positive elliptic systems from [12].

The outline of the paper is as follows. In Section 2, we present the mathematical setting of the vibration problem for a laminated plate. The resulting spectral problem is shown to be well posed. The eigenvalues and eigenfunctions are shown to converge to the corresponding ones of the limit problem as the thickness of the laminated plate goes to zero, which corresponds to a Kirchhoff laminated plate. The finite element discretization is introduced in Section 3 and optimal orders of convergence are proved. These estimates are proved to be independent of the plate thickness and this allows us to conclude that the method is locking-free. In Section 4, we report several numerical tests confirming the theoretical results and showing the good performance of the method. The experiments include some cases not covered by the theoretical analysis, where optimal orders of convergence are also attained. Finally, we prove in the Appendix a thickness-independent a priori estimate for the regularity of the solution of the corresponding load problem.

Throughout the paper, we will use standard notation for Sobolev and Lebesgue spaces. Moreover, $\|\cdot\|_{0}$ will denote the standard norm of $L^{2}(\Omega)$ (or $L^{2}(\Omega)^{n}$, as corresponds). Analogously, $\|\cdot\|_{k}$ will denote the norm of $H^{k}(\Omega)\left(\right.$ or $\left.H^{k}(\Omega)^{n}\right)$. Finally, $C$ will denote a generic constant, not necessarily the same at each occurrence, but always independent of the plate thickness $t$, the particular functions involved, and, in Section 3, also independent of the mesh-size. 


\section{Reissner-Mindlin laminated Plate Equations}

Consider an elastic plate of thickness $t$ with reference configuration $\Omega \times\left(-\frac{t}{2}, \frac{t}{2}\right)$, where $\Omega \subset \mathbb{R}^{2}$ is a convex polygonal domain. The plate is made of two different materials, one occupying the subdomain $\Omega \times\left(-\frac{t}{2}, 0\right)$ and the other one $\Omega \times\left(0, \frac{t}{2}\right)$.

According to the Reissner-Mindlin model, the plate deformation is described by means of the in-plane and transverse displacements, $u^{*}=\left(u_{1}^{*}, u_{2}^{*}\right)$ and $w^{*}$, respectively, and the rotations $\beta^{*}=\left(\beta_{1}^{*}, \beta_{2}^{*}\right)$ of its mid-surface $\Omega$. For the forthcoming analysis, we assume that the plate is clamped on its whole boundary $\partial \Omega$.

The vibration problem for such a plate can be formally obtained from the three-dimensional linear elasticity equations as follows: According to the ReissnerMindlin hypotheses, the admissible displacements at each point are given by

$$
\left(u^{*}(x)-x_{3} \beta^{*}(x), w^{*}(x)\right), \quad x:=\left(x_{1}, x_{2}\right) \in \Omega, \quad x_{3} \in\left(-\frac{t}{2}, \frac{t}{2}\right) .
$$

Test and trial displacements of this form are taken in the variational formulation of the linear elasticity equations for the vibration problem of the three-dimensional plate. By integrating over the thickness and multiplying the shear term by a correcting factor, one arrives at the following problem (see [16]):

Find $\omega>0$ and nontrivial $\left(u^{*}, \beta^{*}, w^{*}\right) \in V$ satisfying

$$
\begin{aligned}
t\left(\mathcal{A} \varepsilon\left(u^{*}\right), \varepsilon(v)\right)+t^{2}\left[\left(\mathcal{B} \varepsilon\left(u^{*}\right), \varepsilon(\eta)\right)+\left(\mathcal{B} \varepsilon\left(\beta^{*}\right), \varepsilon(v)\right)\right] \\
+t^{3}\left(\mathcal{D} \varepsilon\left(\beta^{*}\right), \varepsilon(\eta)\right)+t \kappa\left(\beta^{*}-\nabla w^{*}, \eta-\nabla z\right) \\
=\omega^{2}\left\{\frac{t}{2}\left(\rho_{1}+\rho_{2}\right)\left(u^{*}, v\right)+\frac{t^{3}}{24}\left(\rho_{1}+\rho_{2}\right)\left(\beta^{*}, \eta\right)\right. \\
\left.\quad+\frac{t}{2}\left(\rho_{1}+\rho_{2}\right)\left(w^{*}, z\right)+\frac{t^{2}}{8}\left(\rho_{1}-\rho_{2}\right)\left[\left(\beta^{*}, v\right)+\left(u^{*}, \eta\right)\right]\right\} \quad \forall(v, \eta, z) \in V,
\end{aligned}
$$

where

$$
V:=H_{0}^{1}(\Omega)^{2} \times H_{0}^{1}(\Omega)^{2} \times H_{0}^{1}(\Omega) .
$$

In the equation above $\omega$ is the angular vibration frequency, $(\cdot, \cdot)$ denotes the standard $L^{2}(\Omega)$ inner product of scalar, vector or tensor fields, as corresponds, and $\varepsilon$ is the linear strain tensor defined by $\varepsilon_{i j}(v):=\frac{1}{2}\left(\partial v_{i} / \partial x_{j}+\partial v_{j} / \partial x_{i}\right), i, j=1,2$. Moreover, $\mathcal{A}, \mathcal{B}$, and $\mathcal{D}$ are fourth-order tensors defined by

$$
\mathcal{A}(\tau):=\frac{1}{2}\left(\mathcal{C}_{1}+\mathcal{C}_{2}\right) \tau, \quad \mathcal{B}(\tau):=\frac{1}{8}\left(\mathcal{C}_{1}-\mathcal{C}_{2}\right) \tau, \quad \text { and } \quad \mathcal{D}(\tau):=\frac{1}{24}\left(\mathcal{C}_{1}+\mathcal{C}_{2}\right) \tau
$$

where $\mathcal{C}_{1}$ and $\mathcal{C}_{2}$ are the linear elasticity operators on each medium,

$$
\mathcal{C}_{i} \tau:=\lambda_{i} \operatorname{tr}(\tau) I+2 \mu_{i} \tau, \quad i=1,2,
$$

with plane stress Lamé coefficients $\lambda_{i}:=E_{i} \nu_{i} /\left(1-\nu_{i}^{2}\right)$ and $\mu_{i}:=E_{i} /\left[2\left(1+\nu_{i}\right)\right]$, $E_{i}$ being the Young modulus and $\nu_{i}$ the Poisson ratio of each material. Finally, $\kappa:=k\left(\mu_{1}+\mu_{2}\right) / 2$ is the shear modulus of the laminated plate, with $k$ an appropriate correction factor, and $\rho_{i}$ is the density of each material.

We rescale the problem using new variables

$$
u:=u^{*} / t, \quad \beta:=\beta^{*}, \quad w=: w^{*}, \quad \text { and } \quad \lambda_{t}:=\frac{\rho_{1}+\rho_{2}}{2} \frac{\omega^{2}}{t^{2}} .
$$

The reason for this is that the rescaled variables attain finite nonzero limits as $t$ goes to zero, as will be shown below (cf. Lemma 2.2). We also introduce the scaled 
shear stress

$$
\gamma:=\frac{\kappa}{t^{2}}(\beta-\nabla w)
$$

and the bilinear forms

$$
a((u, \beta),(v, \eta)):=(\mathcal{A} \varepsilon(u), \varepsilon(v))+(\mathcal{B} \varepsilon(u), \varepsilon(\eta))+(\mathcal{B} \varepsilon(v), \varepsilon(\beta))+(\mathcal{D} \varepsilon(\beta), \varepsilon(\eta))
$$

and

$b_{t}((u, \beta, w),(v, \eta, z)):=(w, z)+t^{2}(u, v)+\frac{t^{2}}{12}(\beta, \eta)+\frac{\rho_{1}-\rho_{2}}{4\left(\rho_{1}+\rho_{2}\right)} t^{2}[(\beta, v)+(u, \eta)]$.

Thus, the plate vibration problem can be rewritten as follows:

Find $\lambda_{t}>0$ and nontrivial $(u, \beta, w) \in V$ such that

$$
\left\{\begin{array}{l}
a((u, \beta),(v, \eta))+(\gamma, \eta-\nabla z)=\lambda_{t} b_{t}((u, \beta, w),(v, \eta, z)) \quad \forall(v, \eta, z) \in V \\
\gamma=\frac{\kappa}{t^{2}}(\beta-\nabla w) .
\end{array}\right.
$$

All the eigenvalues $\lambda_{t}$ of this problem are strictly positive, because of the symmetry of both bilinear forms, the ellipticity of $a$, which has been proved in [2, Proposition 2.1], and the positiveness of $b_{t}$, which can be proved by straightforward computations.

To analyze the approximation of this eigenvalue problem, we introduce the operator

$$
T_{t}: H \longrightarrow H
$$

where

$$
H:=L^{2}(\Omega)^{2} \times L^{2}(\Omega)^{2} \times L^{2}(\Omega),
$$

defined for $(f, m, g) \in H$ by $T_{t}(f, m, g):=(u, \beta, w)$, with $(u, \beta, w) \in V$ being the solution to

$$
\left\{\begin{array}{l}
a((u, \beta),(v, \eta))+(\gamma, \eta-\nabla z)=b_{t}((f, m, g),(v, \eta, z)) \quad \forall(v, \eta, z) \in V, \\
\gamma=\frac{\kappa}{t^{2}}(\beta-\nabla w) .
\end{array}\right.
$$

This is the load problem for the Reissner-Mindlin laminated clamped plate. It is a well-posed problem; in fact, the existence and uniqueness of the solution for all $t>0$ follows from [2, Proposition 2.1].

Because of the symmetry of the bilinear forms $a$ and $b_{t}$, the operator $T_{t}$ is selfadjoint in $H$ endowed with the inner-product $b_{t}(\cdot, \cdot)$. The norm induced by this inner product is equivalent to the weighted $L^{2}$ norm

$$
|(v, \eta, z)|_{t}^{2}:=t^{2}\|v\|_{0}^{2}+t^{2}\|\eta\|_{0}^{2}+\|z\|_{0}^{2}, \quad(v, \eta, z) \in H
$$

with equivalence constants independent of $t$. On the other hand, because of the compact embedding $H_{0}^{1}(\Omega) \hookrightarrow L^{2}(\Omega), T_{t}$ is a compact operator. Then, apart from 0 , its spectrum consists of a sequence of finite multiplicity real eigenvalues converging to zero. Note that $\lambda_{t}$ is an eigenvalue of problem (2.1) if and only if $\mu_{t}:=1 / \lambda_{t}$ is an eigenvalue of $T_{t}$, with the same multiplicity and corresponding eigenfunctions.

The solution of the load problem (2.2) satisfies the following additional regularity result, which is systematically used in the proofs that follow: $u, \beta \in H^{2}(\Omega)^{2}, w \in$ $H^{2}(\Omega), \gamma \in H^{1}(\Omega)^{2}$, and there exists a constant $C>0$, independent of $t$ and $(f, m, g)$, such that

$$
\|u\|_{2}+\|\beta\|_{2}+\|w\|_{2}+\|\gamma\|_{0}+t\|\gamma\|_{1} \leq C\left(t^{2}\|f\|_{0}+t^{2}\|m\|_{0}+\|g\|_{0}\right) .
$$


The proof of this a priori estimate is far from being straightforward. In fact, although it is proved by extending similar arguments used for classical homogeneous plates in [1, Theorem 7.1], it needs some preliminary results. Thus, for the sake of clarity, we postpone this analysis to the Appendix. In particular, the estimate (2.3) is a consequence of Theorem 6.5 from this Appendix (cf. Corollary 6.6).

On the other hand, as a consequence of [7, Theorem 1], it can be shown as in 2. Proposition 2.2] that, when $t \rightarrow 0$, the solution $(u, \beta, w)$ to (2.2) converges to $\left(u_{0}, \nabla w_{0}, w_{0}\right)$, where $\left(u_{0}, w_{0}\right) \in H_{0}^{1}(\Omega)^{2} \times H_{0}^{2}(\Omega)$ satisfies

$$
a\left(\left(u_{0}, \nabla w_{0}\right),(v, \nabla z)\right)=(g, z) \quad \forall(v, z) \in H_{0}^{1}(\Omega)^{2} \times H_{0}^{2}(\Omega) .
$$

This limit problem is well posed and corresponds to the bending of a clamped Kirchhoff-type laminated plate subjected to a (rescaled) transverse load $g$. The arguments from [6, Section VII.3.1] can be easily adapted to prove that the limit problem above is equivalent to finding $\left(u_{0}, \beta_{0}, w_{0}\right) \in V$ such that there exists $\gamma_{0} \in$ $H_{0}(\operatorname{rot}, \Omega)^{\prime}$ satisfying

$$
\begin{cases}a\left(\left(u_{0}, \beta_{0}\right),(v, \eta)\right)+\left\langle\gamma_{0}, \eta-\nabla z\right\rangle=b_{0}((f, m, g),(v, \eta, z)) \equiv(g, z) & \forall(v, \eta, z) \in V, \\ \beta_{0}-\nabla w_{0}=0, & \end{cases}
$$

where $\langle\cdot, \cdot\rangle$ stands for the duality pairing in

$$
H_{0}(\operatorname{rot}, \Omega):=\left\{\psi \in L^{2}(\Omega)^{2}: \operatorname{rot} \psi \in L^{2}(\Omega) \text { and } \psi \cdot \tau=0 \text { on } \partial \Omega\right\},
$$

with $\operatorname{rot} \psi:=\partial_{1} \psi_{2}-\partial_{2} \psi_{1}$ and $\tau$ being a unit vector tangent to $\partial \Omega$. Moreover, the arguments from [6] can also be adapted to prove that this is a well-posed mixed problem. Thus, we are allowed to introduce the operator

$$
T_{0}: H \longrightarrow H,
$$

defined for $(f, m, g) \in H$ by $T_{0}(f, m, g):=\left(u_{0}, \beta_{0}, w_{0}\right)$, with $\left(u_{0}, \beta_{0}, w_{0}\right)$ being the solution to problem (2.4).

An a priori estimate similar to (2.3) holds for the limit problem (2.4), as well; namely, $u_{0}, \beta_{0} \in H^{2}(\Omega)^{2}, w_{0} \in H^{2}(\Omega), \gamma_{0} \in L^{2}(\Omega)^{2}$, and

$$
\left\|u_{0}\right\|_{2}+\left\|\beta_{0}\right\|_{2}+\left\|w_{0}\right\|_{2}+\left\|\gamma_{0}\right\|_{0} \leq C\|g\|_{0} .
$$

The proof of this estimate is a consequence of Lemma 6.2 from the Appendix (cf. Corollary 6.3).

Now, we may proceed as in [9] to prove an estimate for the convergence of the solution to (2.2) to that of (2.4) as $t$ goes to zero. In fact, we have the following result.

Lemma 2.1. There exists a constant $C>0$, independent of $t$, such that

$$
\left\|\left(T_{t}-T_{0}\right)(f, m, g)\right\|_{1} \leq C t|(f, m, g)|_{t} \quad \forall(f, m, g) \in H .
$$

Proof. Repeating the arguments of the proof of Lemma 6.4 from the Appendix we arrive at

$$
\left\|u-u_{0}\right\|_{1}+\left\|\beta-\beta_{0}\right\|_{1} \leq C t\|(f, m, g)\|_{0}
$$

(cf. (6.19)). Next, subtracting the second equation in (2.4) from that in (2.2), we have

$$
\gamma=\frac{\kappa}{t^{2}}\left(\beta-\beta_{0}-\nabla\left(w-w_{0}\right)\right) .
$$


Hence, the estimate above and (2.3) yield

$$
\left\|w-w_{0}\right\|_{1} \leq \frac{t^{2}}{\kappa}\|\gamma\|_{0}+\left\|\beta-\beta_{0}\right\|_{0} \leq C t|(f, m, g)|_{b_{t}} .
$$

Thus, we conclude the proof.

As a consequence of this lemma, the operator $\left.T_{t}\right|_{V}$ converges in norm to $\left.T_{0}\right|_{V}$. Then, standard properties of separation of isolated parts of the spectrum (see for instance [11]) yield the following result.

Lemma 2.2. Let $\mu_{0}>0$ be an eigenvalue of $T_{0}$ of multiplicity $m$. Let $D$ be any disc in the complex plane centered at $\mu_{0}$ and containing no other element of the spectrum of $T_{0}$. Then, for $t$ small enough, $D$ contains exactly $m$ eigenvalues of $T_{t}$ (repeated according to their respective multiplicities). Consequently, each eigenvalue $\mu_{0}>0$ of $T_{0}$ is a limit of eigenvalues $\mu_{t}$ of $T_{t}$, as $t$ goes to zero.

\section{Finite-Element discretization}

We restrict our analysis to the DL3 elements introduced in [10, although other finite elements can be analyzed in this same framework. We consider a regular family of triangulations $\left\{\mathcal{T}_{h}\right\}$; as usual, $h$ denotes the mesh-size.

To discretize the rotations, we use standard piecewise linear functions augmented in such a way that they have quadratic tangential components on the boundary of each element. More precisely, for each $T \in \mathcal{T}_{h}$, let $\alpha_{i}^{T}, i=1,2,3$, be its barycentric coordinates and $\tau_{i}^{T}$ a unit vector tangent to the edge $\alpha_{i}^{T}=0$. Consider the edgebubble vector fields $\varphi_{i}^{T}:=\alpha_{j}^{T} \alpha_{k}^{T} \tau_{i}^{T}, i, j, k=1,2,3$, all different. The finite element space for the rotations is defined by

$$
X_{h}:=\left\{\eta_{h} \in H_{0}^{1}(\Omega)^{2}:\left.\eta_{h}\right|_{T} \in \mathcal{P}_{1}^{2} \oplus\left\langle\varphi_{1}^{T}, \varphi_{2}^{T}, \varphi_{3}^{T}\right\rangle \quad \forall T \in \mathcal{T}_{h}\right\} .
$$

We use standard piecewise linear elements for the displacements; namely,

$$
W_{h}:=\left\{z_{h} \in H_{0}^{1}(\Omega):\left.z_{h}\right|_{T} \in \mathcal{P}_{1}, \forall T \in \mathcal{T}_{h}\right\}
$$

for the transverse displacements and

$$
U_{h}:=W_{h}^{2}
$$

for the in-plane displacements. Thus, the finite element discretization of the space $V$ is defined by

$$
V_{h}:=U_{h} \times X_{h} \times W_{h} .
$$

For the numerical method, we also need the so-called reduction operator

$$
R: H^{1}(\Omega)^{2} \cap H_{0}(\operatorname{rot}, \Omega) \longrightarrow \Gamma_{h},
$$

where $\Gamma_{h}$ is the lowest-order rotated Raviart-Thomas space (see [14])

$$
\Gamma_{h}:=\left\{\psi_{h} \in H_{0}(\operatorname{rot}, \Omega):\left.\psi_{h}\right|_{T} \in \mathcal{P}_{0}^{2} \oplus\left(-x_{2}, x_{1}\right) \mathcal{P}_{0} \quad \forall T \in \mathcal{T}_{h}\right\} .
$$

This reduction operator is uniquely determined by

$$
\int_{\ell} R \psi \cdot \tau_{\ell}=\int_{\ell} \psi \cdot \tau_{\ell}, \quad \psi \in H^{1}(\Omega)^{2} \cap H_{0}(\operatorname{rot}, \Omega),
$$

for every edge $\ell$ of the triangulation ( $\tau_{\ell}$ being a unit tangent vector along $\ell$ ).

Now we are in a position to write the finite element approximation of the plate vibration problem (2.1): 
Find $\lambda_{t h}>0$ and nontrivial $\left(u_{h}, \beta_{h}, w_{h}\right) \in V_{h}$ such that

$$
\left\{\begin{array}{c}
a\left(\left(u_{h}, \beta_{h}\right),\left(v_{h}, \eta_{h}\right)\right)+\left(\gamma_{h}, R \eta_{h}-\nabla v_{h}\right) \\
=\lambda_{t h} b_{t}\left(\left(u_{h}, \beta_{h}, w_{h}\right),\left(v_{h}, \eta_{h}, z_{h}\right)\right) \quad \forall\left(v_{h}, \eta_{h}, z_{h}\right) \in V_{h}, \\
\gamma_{h}=\frac{\kappa}{t^{2}}\left(R \beta_{h}-\nabla w_{h}\right) .
\end{array}\right.
$$

Notice that the method is nonconforming since consistency terms arise because of the reduction operator.

As in the continuous case, we introduce the operator

$$
T_{t h}: H \longrightarrow H,
$$

defined for $(f, m, g) \in H$ by $T_{t h}(f, m, g):=\left(u_{h}, \beta_{h}, w_{h}\right)$, with $\left(u_{h}, \beta_{h}, w_{h}\right) \in V_{h}$ being the solution to the corresponding finite element discretization of the load problem (2.2), namely,

$$
\left\{\begin{array}{l}
a\left(\left(u_{h}, \beta_{h}\right),\left(v_{h}, \eta_{h}\right)\right)+\left(\gamma_{h}, R \eta_{h}-\nabla z_{h}\right)=b_{t}\left((f, m, g),\left(v_{h}, \eta_{h}, z_{h}\right)\right) \\
\gamma_{h}=\frac{\kappa}{t^{2}}\left(R \beta_{h}-\nabla w_{h}\right) .
\end{array}\right.
$$

The existence and uniqueness of the solution to this problem follows easily from the ellipticity of $a$. Once more $\lambda_{t h}$ is an eigenvalue of problem (3.1) if and only if $\mu_{t h}:=1 / \lambda_{t h}$ is a strictly positive eigenvalue of $T_{t h}$ with the same multiplicity and corresponding eigenfunctions.

For $t>0$ fixed, the spectral approximation theory for compact operators (cf. 4]) can be readily applied to prove convergence of the eigenpairs of $T_{t h}$ to those of $T_{t}$. However, further considerations are needed to show that the error estimates do not deteriorate as $t$ becomes small. With this goal, we will make use of the following result, which will lead to optimal error estimates in the $H^{1}$ norm for displacements and rotations.

Lemma 3.1. There exists a constant $C$, independent of $t$ and $h$, such that

$$
\left\|\left(T_{t}-T_{t h}\right)(f, m, g)\right\|_{1} \leq C h|(f, m, g)|_{t} \quad \forall(f, m, g) \in H .
$$

Proof. Let $(f, m, g) \in H,(u, \beta, w):=T_{t}(f, m, g)$, and $\left(u_{h}, \beta_{h}, w_{h}\right):=T_{t h}(f, m, g)$. The arguments given in the proof of [2, Proposition 3.2] for pure transverse loads (i.e., $f=0$ and $m=0$ ) extend easily to our case yielding

$$
\begin{aligned}
\left\|u-u_{h}\right\|_{1}+\left\|\beta-\beta_{h}\right\|_{1}+ & \left\|w-w_{h}\right\|_{1}+t\left\|\gamma-\gamma_{h}\right\|_{0} \\
& \leq C h\left(\|u\|_{2}+\|\beta\|_{2}+\|\gamma\|_{0}+t\|\gamma\|_{1}\right) .
\end{aligned}
$$

Thus, the lemma follows from this inequality and (2.3).

As a consequence of this lemma, $\left.T_{t h}\right|_{V}$ converges in norm to $\left.T_{t}\right|_{V}$. In fact, for any fixed $t \in\left(0, t_{\max }\right)$, we have $|\cdot|_{t} \leq t_{\max }\|\cdot\|_{1}$ and hence the lemma yields

$$
\left\|\left(T_{t}-T_{t h}\right)(f, m, g)\right\|_{1} \leq C h\|(f, m, g)\|_{1} \quad \forall(f, m, g) \in V .
$$

Consequently, if $\mu_{t}$ is an eigenvalue of $T_{t}$ with multiplicity $m$, then exactly $m$ eigenvalues of $T_{t h}$ (repeated according to their respective multiplicities) converge to $\mu_{t}$ as $h$ goes to zero (see [11). The following theorem shows that, under mild assumptions, optimal $t$-independent error estimates in the $H^{1}$ norm are valid for the eigenfunctions. 
Theorem 3.2. Let $\mu_{t}$ be an eigenvalue of $T_{t}$ converging to a simple eigenvalue $\mu_{0}$ of $T_{0}$, as $t$ goes to zero. Let $\mu_{t h}$ be the eigenvalue of $T_{\text {th }}$ that converges to $\mu_{t}$ as $h$ goes to zero. Let $(u, \beta, w)$ and $\left(u_{h}, \beta_{h}, w_{h}\right)$ be the corresponding eigenfunctions conveniently normalized. Then, for $t$ and $h$ small enough,

$$
\left\|(u, \beta, w)-\left(u_{h}, \beta_{h}, w_{h}\right)\right\|_{1} \leq C h,
$$

with a constant $C$ independent of $t$ and $h$.

Proof. The inequality of the theorem is a direct consequence of the estimate (3.2) and [4, Theorem 7.1], with a constant $C$ depending on the constant in (3.2) (which is independent of $t$ ) and on the inverse of the distance of $\mu_{t}$ to the rest of the spectrum of $T_{t}$. Now, according to Lemma 2.2. for $t$ small enough, this distance can be bounded below in terms of the distance of $\mu_{0}$ to the rest of the spectrum of $T_{0}$, which obviously does not depend on $t$. Thus, we conclude the proof.

The following lemma is the basic tool to prove a double order of convergence for the eigenvalues.

Lemma 3.3. There exists a constant $C$, independent of $t$ and $h$, such that

$$
\left\|\left(T_{t}-T_{t h}\right)(f, m, g)\right\|_{0} \leq C h^{2}|(f, m, g)|_{t} \quad \forall(f, m, g) \in H .
$$

Proof. We do not include it, since it is a straightforward modification of the proof of [9, Lemma 3.4].

Theorem 3.4. Let $\mu_{t}$ and $\mu_{t h}$ be as in Theorem 3.2. Then, for $t$ and $h$ small enough,

$$
\left|\mu_{t}-\mu_{t h}\right| \leq C h^{2}
$$

with a constant $C$ independent of $t$ and $h$.

Proof. Let $(u, \beta, w)$ be an eigenfunction corresponding to $\mu_{t}$ normalized in the norm induced by $b_{t}$. Applying [4, Theorem 7.3] and taking into account that $T$ and $T_{h}$ are self-adjoint with respect to $b_{t}$, we have

$$
\left|\mu_{t}-\mu_{t h}\right| \leq C\left[b_{t}\left(\left(T_{t}-T_{t h}\right)(u, \beta, w),(u, \beta, w)\right)+\left|\left(T_{t}-T_{t h}\right)(u, \beta, w)\right|_{t}^{2}\right],
$$

with a constant $C$ depending on the inverse of the distance of $\mu_{t}$ to the rest of the spectrum of $T_{t}$. By repeating the arguments in the proof of Theorem 3.2 we observe that, for $t$ small enough, this constant can be chosen independent of $t$. Thus, since $|\cdot|_{t} \leq C\|\cdot\|_{0}$, using the estimate from Lemma 3.3 in (3.3), we conclude the proof.

Another consequence of Lemma 3.3 is a double order of convergence for the eigenfunctions in the $L^{2}$ norm.

Theorem 3.5. Let $\mu_{t}, \mu_{t h},(u, \beta, w)$ and $\left(u_{h}, \beta_{h}, w_{h}\right)$ be as in Theorem 3.2. Then, for $t$ and $h$ small enough,

$$
\left\|(u, \beta, w)-\left(u_{h}, \beta_{h}, w_{h}\right)\right\|_{0} \leq C h^{2},
$$

with a constant $C$ independent of $t$ and $h$.

Proof. Since $|\cdot|_{t} \leq C\|\cdot\|_{0}$, the arguments in the proof of Theorem 3.2 can be repeated using $\|\cdot\|_{0}$ instead of $\|\cdot\|_{1}$ and the estimate from Lemma 3.3 instead of (3.2). 


\section{NUMERICAL EXPERIMENTS}

We report in this section some numerical results obtained with a code which implements the method analyzed above. The aim of this numerical experimentation is two-fold: to confirm the theoretical results and to assess the performance of the method.

4.1. Test 1: A simply supported rectangular plate with a known analytical solution. Validation. The aim of this first test is to validate the computer code and to corroborate the error estimates proved in the previous section. With this purpose, we applied the method to a problem with a known analytical solution: a rectangular simply supported plate (see [15, 16]). This test allowed us to calculate the error of the different quantities computed with our code. Moreover, it shows that the method is able to deal with other kinds of boundary conditions, although the theoretical analysis has been made just for clamped plates.

If the domain of the simply supported plate is the rectangle $\Omega:=(0, a) \times(0, b)$, then the eigenfunctions are given by

$$
u=\left[\begin{array}{l}
\hat{u}_{1} \cos \frac{k \pi x}{a} \sin \frac{l \pi y}{b} \\
\hat{u}_{2} \sin \frac{k \pi x}{a} \cos \frac{l \pi y}{b}
\end{array}\right], \quad w=\hat{w} \sin \frac{k \pi x}{a} \sin \frac{l \pi y}{b}, \quad=\left[\begin{array}{l}
\hat{\beta}_{1} \cos \frac{k \pi x}{a} \sin \frac{l \pi y}{b} \\
\hat{\beta}_{2} \sin \frac{k \pi x}{a} \cos \frac{l \pi y}{b}
\end{array}\right],
$$

$k, l \in \mathbb{N}$. The constants $\hat{u}_{1}, \hat{u}_{2}, \hat{w}, \hat{\beta}_{1}$, and $\hat{\beta}_{2}$, as well as the corresponding eigenvalues, can be obtained as follows: For each pair $(k, l) \in \mathbb{N}^{2}$, the terms (4.1) must be plugged into (2.1), written in strong form. This leads to a $5 \times 5$ generalized eigenvalue problem whose eigenvectors are $\left(\hat{u}_{1}, \hat{u}_{2}, \hat{w}, \hat{\beta}_{1}, \hat{\beta}_{2}\right)^{\mathrm{t}}$ and whose eigenvalues are the ones we are looking for.

We applied the method to a plate of length $a=6 \mathrm{~m}$, width $b=4 \mathrm{~m}$, and thickness $t=0.1 \mathrm{~m}$. We used the following physical parameters:

$$
\begin{array}{lll}
E_{1}=1.440 \times 10^{11} \mathrm{~N} / \mathrm{m}^{2}, & \nu_{1}=0.35, & \rho_{1}=7700 \mathrm{~kg} / \mathrm{m}^{3}, \\
E_{2}=0.144 \times 10^{11} \mathrm{~N} / \mathrm{m}^{2}, & \nu_{2}=0.30, & \rho_{2}=770 \mathrm{~kg} / \mathrm{m}^{3} .
\end{array}
$$

Finally, we took $k=5 / 6$ as a correction factor for this and all the other tests.

We used uniform meshes obtained by refining the coarse one shown in Figure 1]. The refinement parameter $N$ is the number of layers of elements through the width of the plate.

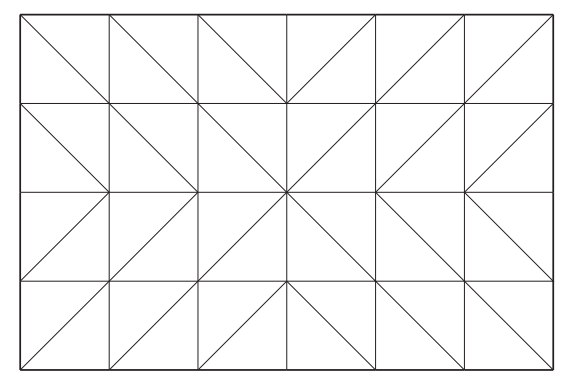

Figure 1. Rectangular plate. Finite element coarse mesh $(N=4)$.

Table 1 shows the six lowest vibration frequencies computed with the method on four successively refined meshes. The table also includes the corresponding exact 
values obtained from the analytical solution and the computed order of convergence for each one.

TABle 1. Test 1: Lowest vibration frequencies of a simply supported laminated rectangular plate.

\begin{tabular}{crrrrrc}
\hline Mode & $N=8$ & $N=16$ & $N=32$ & $N=64$ & Exact & Order \\
\hline$\omega_{1}$ & 84.605 & 83.368 & 83.066 & 82.992 & 82.967 & 2.02 \\
$\omega_{2}$ & 166.169 & 161.063 & 159.829 & 159.527 & 159.427 & 2.03 \\
$\omega_{3}$ & 272.770 & 259.194 & 255.911 & 255.102 & 254.834 & 2.02 \\
$\omega_{4}$ & 308.314 & 291.870 & 287.894 & 286.918 & 286.595 & 2.02 \\
$\omega_{5}$ & 361.027 & 338.177 & 332.772 & 331.458 & 331.026 & 2.04 \\
$\omega_{6}$ & 514.209 & 471.247 & 461.043 & 458.564 & 457.749 & 2.04 \\
\hline
\end{tabular}

A quadratic order of convergence can be clearly observed for all the vibration frequencies, which corresponds to an optimal double order according to the degree of the finite elements used.

Figure 2 shows the error curves in the $L^{2}$ norm and the $H^{1}$ seminorm of the inplane and the transverse displacements, $u$ and $w$, respectively, for the eigenfunction corresponding to the lowest vibration frequency $\omega_{1}$.
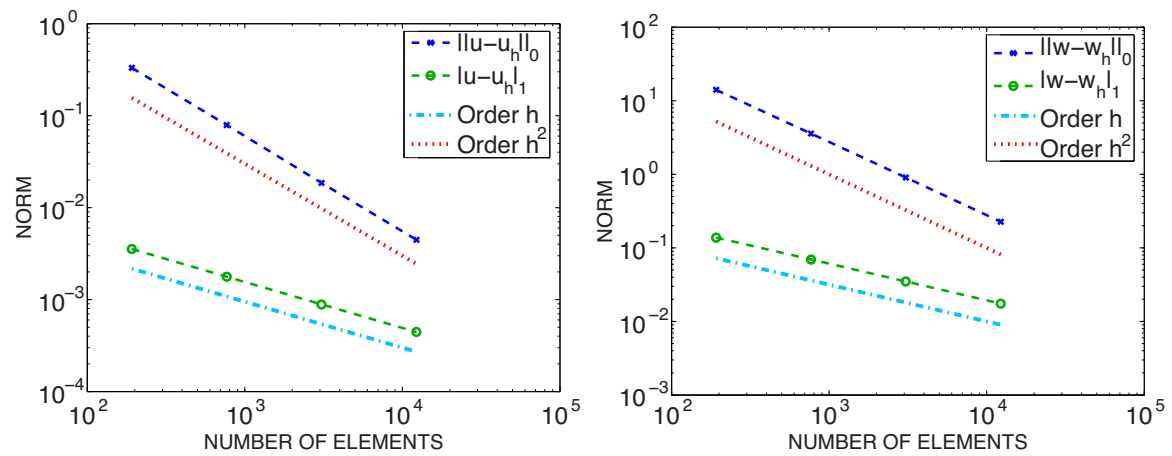

Figure 2. Test 1: Error curves for in-plane displacements (left) and transverse displacements (right); log-log plots of the corresponding norms versus the number of elements.

A quadratic order in $L^{2}$ and a linear order in $H^{1}$ can be clearly observed for both displacements. Once more, this corresponds to the optimal orders according to the degree of the finite elements.

4.2. Test 2: A clamped rectangular plate. Testing the locking-free character of the method. The main goal of this test is to confirm experimentally that the method is locking-free, as was proved in the previous section. With this purpose, we chose a problem lying in the theoretical framework: a plate clamped on its whole boundary.

We used a rectangular plate with the same dimensions and physical parameters as in the previous test. First, we also used the same meshes. 
We computed the lowest vibration frequencies of the plate on each mesh. Since no analytical solution is available in this case, for each vibration mode, we extrapolated a more accurate approximation of the frequency and estimated the order of convergence by means of a least square fitting.

Table 2 shows the six lowest vibration frequencies computed on different meshes, the estimated order of convergence and the extrapolated more accurate value of each frequency.

TABLE 2. Test 2: Lowest vibration frequencies of a clamped laminated rectangular plate computed on uniform meshes.

\begin{tabular}{ccccccc}
\hline Mode & $N=8$ & $N=16$ & $N=32$ & $N=64$ & Order & Extrapolated \\
\hline$\omega_{1}$ & 161.136 & 157.902 & 157.095 & 156.898 & 2.01 & 156.831 \\
$\omega_{2}$ & 255.847 & 245.336 & 242.757 & 242.128 & 2.03 & 241.924 \\
$\omega_{3}$ & 418.592 & 391.593 & 384.980 & 383.358 & 2.03 & 382.835 \\
$\omega_{4}$ & 419.684 & 393.806 & 387.352 & 385.762 & 2.01 & 385.237 \\
$\omega_{5}$ & 518.006 & 475.120 & 464.831 & 462.331 & 2.06 & 461.574 \\
$\omega_{6}$ & 667.968 & 603.579 & 587.688 & 583.781 & 2.02 & 582.499 \\
\hline
\end{tabular}

Once more, a double order of convergence can be readily observed for all the vibration frequencies.

In the previous tests we have used uniform meshes. In what follows we will show that the performance of the method is essentially the same on nonuniform meshes. With this purpose, we have solved the same problem with tiled meshes as those shown in Figure 3. the reason for this choice is to avoid asymptotically uniform meshes. The refinement parameter $M$ used this time to label each mesh is the number of tiles on each vertical edge of the plate.
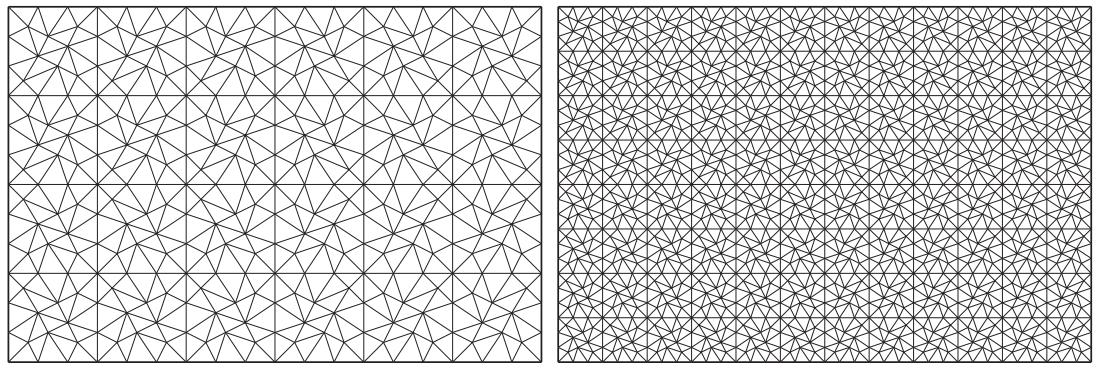

Figure 3. Rectangular plate. Tiled meshes: $M=1$ (left), $M=2$ (right)

Table 3 shows results analogous to those from Table 2, but computed with this kind of meshes. No significant difference can be observed between the results on uniform and nonuniform meshes. The order of convergence is clearly quadratic in both cases and the size of the error depends essentially on the number of elements and not on the type of mesh.

Next, we tested whether the method remains locking-free as the thickness becomes small. For this test, we took clamped plates with the same physical parameters and dimensions as above, except for the thickness for which we used different values ranging from $t=0.1 \mathrm{~m}$ to $0.1 \mathrm{~mm}$. 
TABLE 3. Test 2: Lowest vibration frequencies of a clamped laminated rectangular plate computed on nonuniform meshes.

\begin{tabular}{ccccccc}
\hline Mode & $M=1$ & $M=2$ & $M=3$ & $M=4$ & Order & Extrapolated \\
\hline$\omega_{1}$ & 158.534 & 157.247 & 157.015 & 156.935 & 2.04 & 156.834 \\
$\omega_{2}$ & 246.352 & 243.013 & 242.401 & 242.189 & 2.02 & 241.920 \\
$\omega_{3}$ & 393.272 & 385.377 & 383.945 & 383.449 & 2.03 & 382.821 \\
$\omega_{4}$ & 396.917 & 388.115 & 386.504 & 385.946 & 2.02 & 385.235 \\
$\omega_{5}$ & 477.505 & 465.444 & 463.240 & 462.477 & 2.02 & 461.501 \\
$\omega_{6}$ & 609.429 & 589.151 & 585.427 & 584.134 & 2.02 & 582.502 \\
\hline
\end{tabular}

To allow for comparison, we report normalized frequencies $\hat{\omega}:=\omega / t$. Table 4 shows the lowest vibration frequency of clamped rectangular laminated plates with decreasing values of the thickness computed on uniform meshes. Once more, the table includes the estimated orders of convergence and extrapolated frequencies. We also report on the last row the extrapolated limit values corresponding to $t=0$ (i.e., the Kirchhoff model).

TABLE 4. Test 2: Normalized lowest frequency $\hat{\omega}_{1}$ of clamped laminated rectangular plates with varying thickness computed on uniform meshes.

\begin{tabular}{lcccccc}
\hline Thickness & $N=8$ & $N=16$ & $N=24$ & $N=32$ & Order & Extrapolated \\
\hline$t=0.1 \mathrm{~m}$ & 1611.365 & 1579.017 & 1573.019 & 1570.948 & 2.00 & 1568.239 \\
$t=0.01 \mathrm{~m}$ & 1616.810 & 1584.050 & 1577.875 & 1575.707 & 1.97 & 1572.859 \\
$t=0.001 \mathrm{~m}$ & 1616.866 & 1584.103 & 1577.928 & 1575.759 & 1.97 & 1572.883 \\
$t=0.0001 \mathrm{~m}$ & 1616.865 & 1584.103 & 1577.926 & 1575.762 & 1.97 & 1572.883 \\
\hline$t=0$ (extr.) & 1616.866 & 1584.103 & 1577.927 & 1575.761 & 1.97 & 1572.883 \\
\hline
\end{tabular}

We observe that the method is perfectly locking-free, and that the quadratic order of convergence is preserved even for extremely small values of the thickness.

Results essentially equal to those shown in Table 4 are obtained with the tiled nonuniform meshes shown in Figure 3. We do not include the corresponding table, since there is no significant difference.

4.3. Test 3: A clamped circular plate. Robustness of the plate model and the finite element method. The aim of this test is to assess the efficiency of the Reissner-Mindlin laminated plate model by comparing their results with those obtained from the 3D elasticity equations. In particular, we are interested in exhibiting the robustness of the model, as well as that of the proposed finite element method, when applied to laminates with very different physical parameters. With this purpose, we applied the method to a problem whose corresponding 3D equations can be accurately solved. This is the reason why we chose a circular plate, whose axisymmetric vibration modes can be efficiently computed from the corresponding equations in cylindrical coordinates.

We used a clamped circular plate with diameter $d=2 \mathrm{~m}$ and thickness $t=0.1 \mathrm{~m}$. First, we took the same physical parameters as in the other experiments. We used quasiuniform meshes as that shown in Figure 4. The refinement parameter $N$ is in this case the number of elements lying on a radius of the circle. 


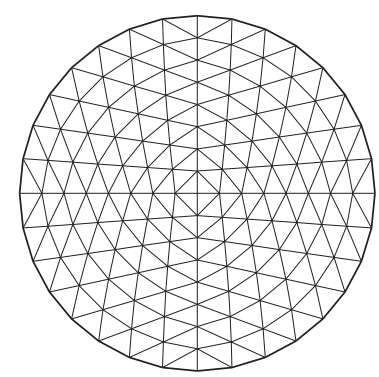

Figure 4. Circular plate. Finite element mesh $(N=8)$.

On the other hand, by taking advantage of the cylindrical symmetry, the threedimensional problem reduces to a two-dimensional one posed on a meridional section of the plate. Thus, we also computed the axisymmetric vibration modes by means of another code based on a standard finite element discretization of the 3D elasticity equations in cylindrical coordinates. Also, in this case we used successively refined meshes and obtained a very accurate approximation of the vibrations frequencies by extrapolation.

We report in Table 5 the results for a couple of axisymmetric modes, which, for this plate, correspond to the first and the sixth vibration frequencies. The table includes again the extrapolated values of the frequencies and the estimated orders of convergence, as well. It also includes in the last column the values obtained with the axisymmetric $3 \mathrm{D}$ code.

TABLE 5. Test 3: Lowest vibration frequencies of axisymmetric modes of a clamped laminated circular plate; Young moduli ratio $E_{1} / E_{2}=10$.

\begin{tabular}{ccrrrrrr}
\hline Mode & $N=4$ & \multicolumn{1}{c}{$N=8$} & \multicolumn{1}{c}{$N=16$} & \multicolumn{1}{c}{$N=32$} & Order & Extrapolated & \multicolumn{1}{c}{$3 \mathrm{D}$} \\
\hline$\omega_{1}$ & 968.270 & 945.923 & 940.395 & 939.020 & 2.01 & 938.571 & 935.748 \\
$\omega_{6}$ & 3908.732 & 3628.509 & 3559.984 & 3543.051 & 2.03 & 3537.664 & 3489.815 \\
\hline
\end{tabular}

It can be seen from this table that the disparity between both extrapolated values is very small indeed, which is merely a confirmation of the accuracy of the Reissner-Mindlin laminated plate model.

Next, we tested the robustness of the model applied to laminates with physical parameters of very different scales. With this aim, we computed the vibration modes of a plate identical to the previous one, except for the fact that the Young modulus of the second material is now $E_{2}=0.144 \times 10^{8} \mathrm{~N} / \mathrm{m}^{2}$. Therefore, the ratio between the Young moduli of each material is $10^{4}$.

We report in Table 6 the results for this plate analogous to those of the previous table.

Once more, an excellent agreement between both models can be clearly observed, despite the disparity of the Young modulus of each material. Other unreported tests demonstrate the robustness of the method for the Reissner-Mindlin laminated plate model with respect to the remaining physical parameters. 
TABLE 6. Test 3: Lowest vibration frequencies of axisymmetric modes of a clamped laminated circular plate; Young moduli ratio $E_{1} / E_{2}=10^{4}$.

\begin{tabular}{crrrrrrr}
\hline Mode & \multicolumn{1}{c}{$N=4$} & \multicolumn{1}{c}{$N=8$} & \multicolumn{1}{c}{$N=16$} & \multicolumn{1}{c}{$N=32$} & Order & Extrapolated & \multicolumn{1}{c}{ 3D } \\
\hline$\omega_{1}$ & 671.490 & 650.794 & 645.813 & 644.582 & 2.05 & 644.206 & 645.057 \\
$\omega_{6}$ & 2810.616 & 2540.776 & 2478.636 & 2463.491 & 2.11 & 2459.352 & 2425.412 \\
\hline
\end{tabular}

Finally, Figures 5 and 6 show the transverse displacement fields computed with the Reissner-Mindlin plate model for the two vibration modes reported in Table 6 . The figures also show the corresponding meridional plate sections of each mode computed with the axisymmetric 3D code.
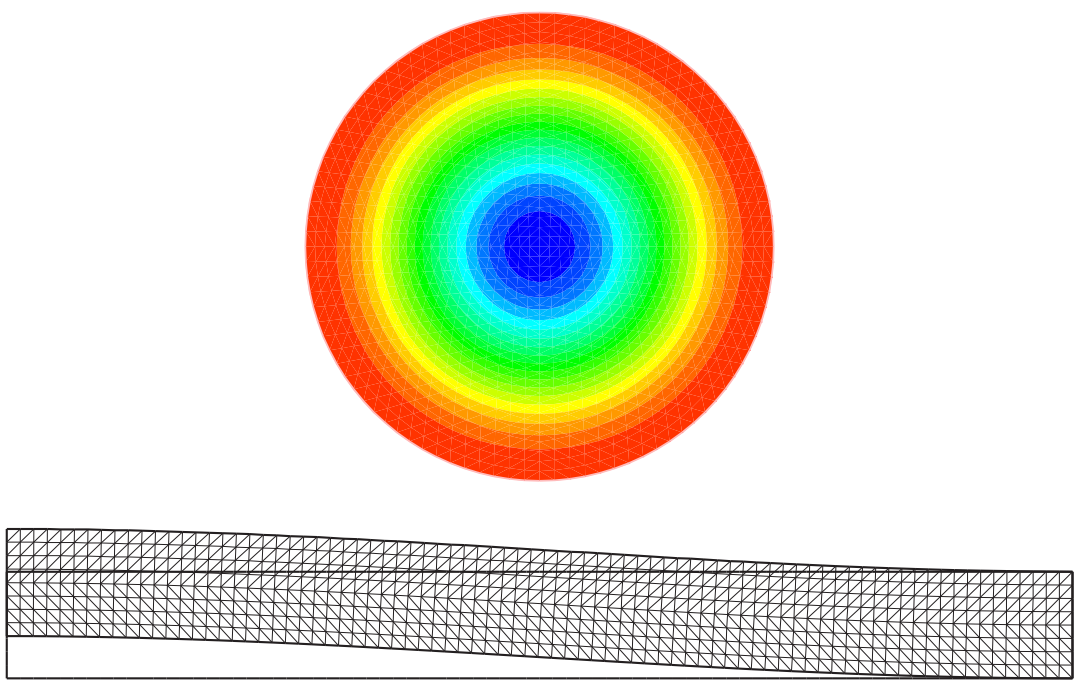

FiguRE 5. Test 3: Lowest axisymmetric vibration mode $\left(\omega_{1}\right)$ of a clamped laminated circular plate with Young moduli ratio $E_{1} / E_{2}=10^{4}$; transverse displacement field (top) and meridional cross section (bottom).

4.4. Test 4: A cantilever rectangular plate. A test with significant coupling between in-plane and transverse displacements. The aim of this test is to assess the performance of the method when there is a significant interplay between the membrane action and the bending behavior of the plate. With this purpose, we applied the method to a cantilever plate, with its dimensions chosen to allow for a significant coupling between in-plane (membrane) and transverse (bending) terms.

In particular, we have used a rectangular plate, with height and width as that from Test 1, with its left edge clamped and all the others free. The thickness of the plate was chosen $t=1 \mathrm{~m}$ so that the frequencies of the lowest membrane modes (i.e., those with in-plane displacements larger than transverse displacements) lie in the range of the lowest bending modes (with transverse displacements larger than 

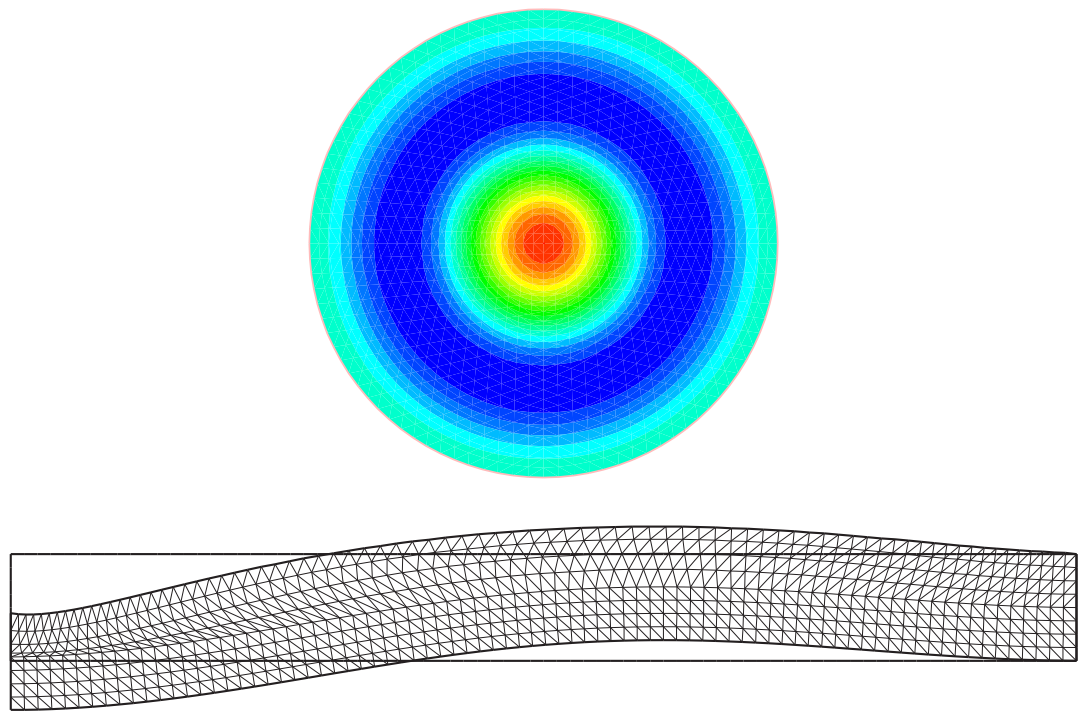

Figure 6. Test 3: Second lowest axisymmetric vibration mode $\left(\omega_{6}\right)$ of a clamped laminated circular plate with Young moduli ratio $E_{1} / E_{2}=10^{4}$; transverse displacement field (top) and meridional cross section (bottom).

in-plane displacements). The physical parameters were taken as follows:

$$
\begin{array}{lll}
E_{1}=1.440 \times 10^{11} \mathrm{~N} / \mathrm{m}^{2}, & \nu_{1}=0.35, & \rho_{1}=7700 \mathrm{~kg} / \mathrm{m}^{3}, \\
E_{2}=1.440 \times 10^{9} \mathrm{~N} / \mathrm{m}^{2}, & \nu_{2}=0.30, & \rho_{2}=770 \mathrm{~kg} / \mathrm{m}^{3} .
\end{array}
$$

The Young moduli ratio $E_{1} / E_{2}=10^{2}$ was taken sufficiently large so that the plate results in an unbalanced laminated structure with a significant coupling between membrane and bending terms.

Figure 7 shows the in-plane and transverse displacements for the four lowest vibration modes. Notice that the third one corresponds to a membrane mode (larger in-plane displacements) whereas the other ones are bending modes (larger transverse displacements). This can be seen more clearly from Figure 8, where the three components of the displacements on the bottom edge of the plate are shown for each mode. The interplay between membrane and bending terms can be seen from the fact that the ratio between transverse and in-plane displacements is, for instance, around 8 for the second vibration mode and around 5 for the fourth one.

Table 7 shows the convergence behavior of the method.

In this case, the orders of convergence are smaller than for clamped and simply supported plates. This behavior agrees with what should be expected from the theoretical results, since the eigenfunctions of these plates have singularities at the vertices of the left edge where the boundary conditions change. Nevertheless, the relative errors are excellent again. In fact, these errors lie below $4 \%$ for the coarsest mesh $(N=8)$, which is a behavior comparable to that for a simply supported or a clamped plate (Tables 1 and 2 respectively). 

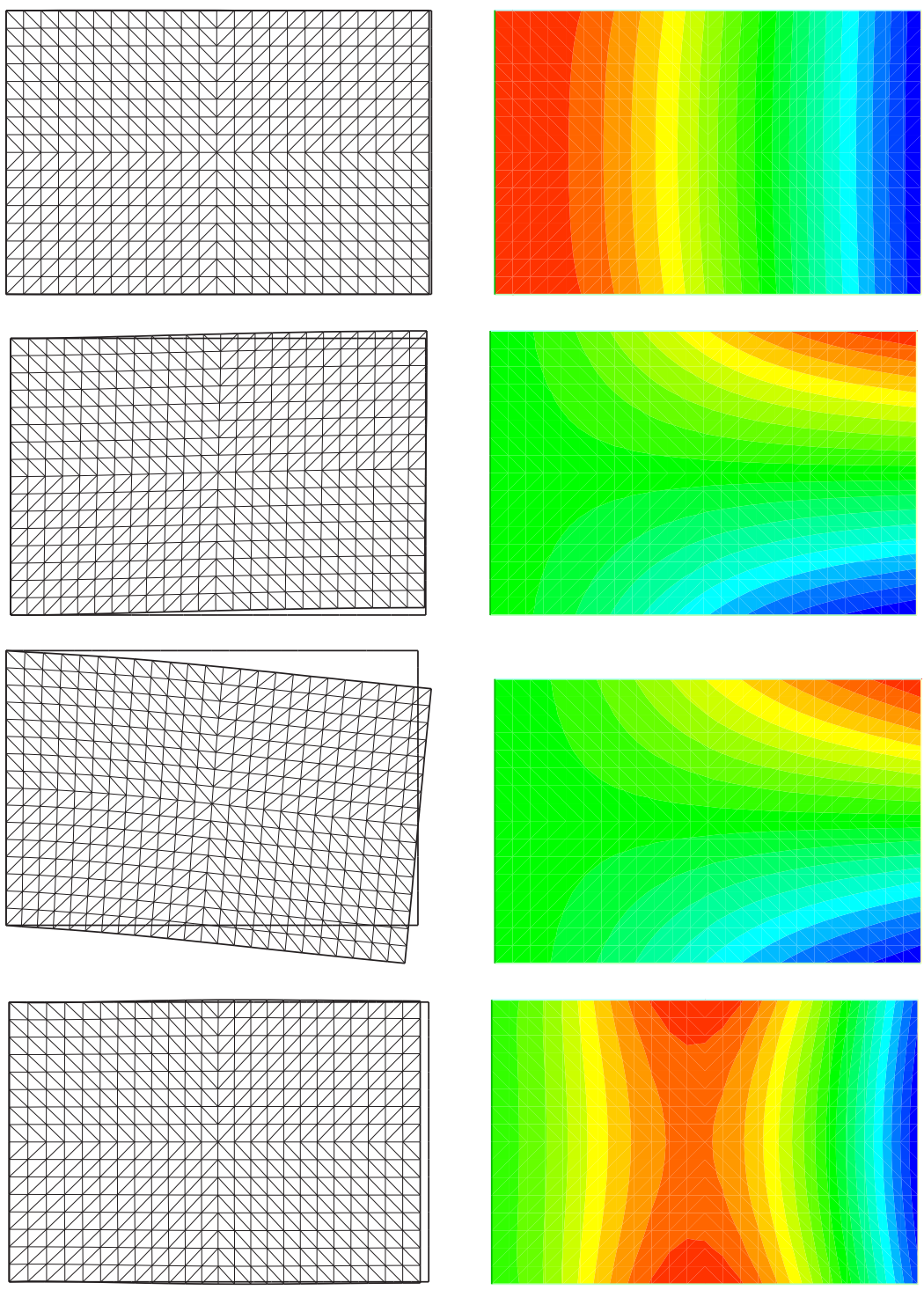

Figure 7. Test 4: Lowest vibration modes of a cantilever laminated rectangular plate.

4.5. Test 5: A simply supported rectangular plate with an eigenvalue cluster. The aim of this test is to investigate numerically the effect of eigenvalue clusters on the convergence of the method.

The vibration frequencies of a simply supported Kirchhoff rectangular laminated plate can also be computed analytically. In fact, a procedure similar to that used in Test 1 works in this case, too. We have used the analytical solution to find a Kirchhoff laminated plate with an eigenvalue with multiplicity two and another one very close to this (relative difference around $0.5 \%$ ). 

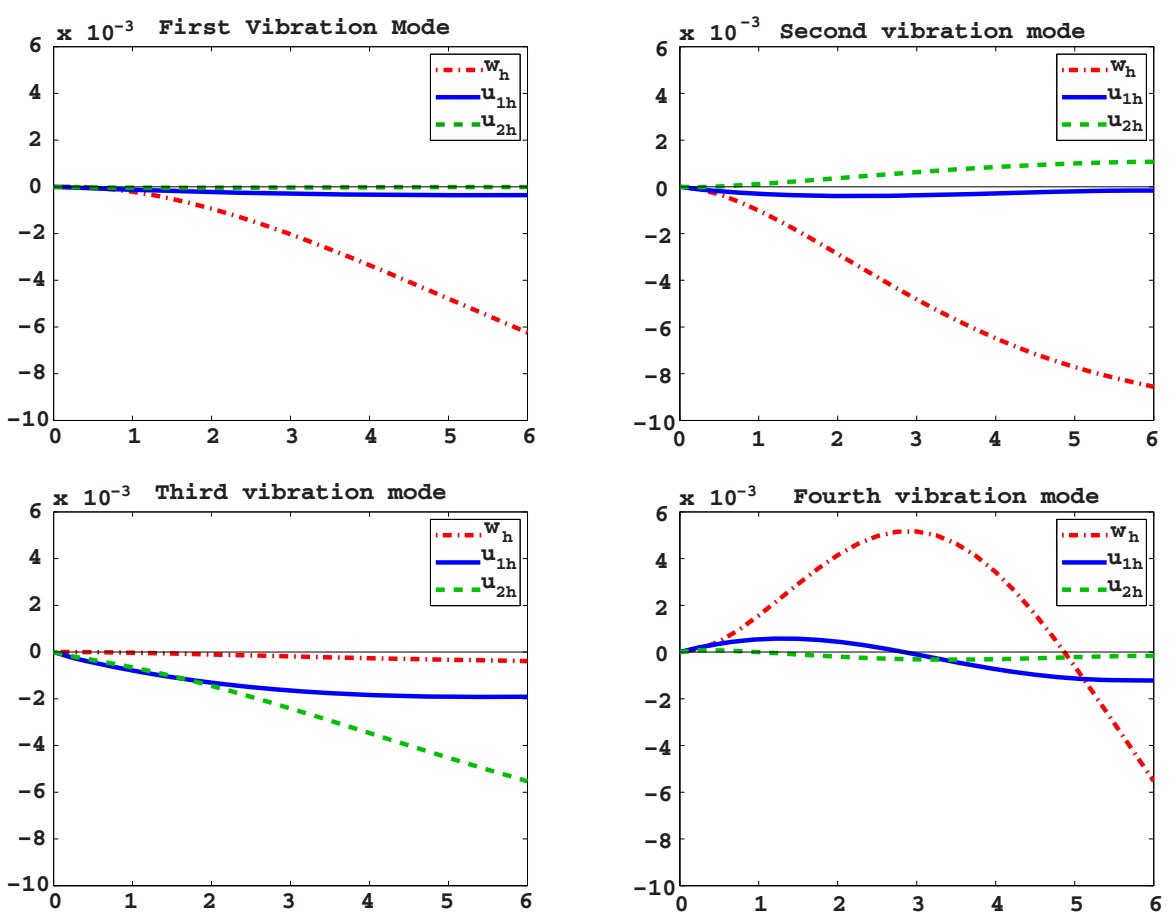

Figure 8. Test 4: Transverse and in-plane displacements along the bottom edge of a cantilever laminated rectangular plate for the lowest vibration modes.

TABLE 7. Test 4: Lowest vibration frequencies of a cantilever laminated rectangular plate.

\begin{tabular}{crrrrcc}
\hline Mode & $N=8$ & $N=16$ & $N=32$ & $N=64$ & Order & Extrapolated \\
\hline$\omega_{1}$ & 63.537 & 63.401 & 63.353 & 63.338 & 1.54 & 63.329 \\
$\omega_{2}$ & 201.077 & 198.273 & 197.191 & 196.861 & 1.45 & 196.626 \\
$\omega_{3}$ & 371.342 & 366.166 & 364.713 & 364.303 & 1.83 & 364.143 \\
$\omega_{4}$ & 381.842 & 377.598 & 376.419 & 376.099 & 1.85 & 375.970 \\
$\omega_{5}$ & 666.090 & 650.996 & 645.859 & 644.351 & 1.60 & 643.490 \\
$\omega_{6}$ & 907.037 & 893.123 & 888.974 & 887.848 & 1.77 & 887.323 \\
\hline
\end{tabular}

Since the double eigenvalue appears not for symmetry reasons, it splits into two different ones in the Reissner-Mindlin model, both converging to it as the thickness becomes smaller. Thus we have a small cluster of three different but very close vibration frequencies, $\omega_{18}, \omega_{19}$, and $\omega_{20}$, in the Reissner-Mindlin plate. These frequencies can also be computed analytically by means of the technique described in Test 1.

The geometrical and physical parameters leading to this cluster are the same as those in Test 1. For the numerical computations, we have used the same uniform meshes as in this test, too. 
We have computed approximations $\omega_{n h}, n=18,19,20$, of the three vibration frequencies in the cluster on the meshes corresponding to $N=16,32,64$, for decreasing values of the thickness $t=0.1,0.01,0.001,0.0001 \mathrm{~m}$.

We have observed that the relative error of each computed vibration frequency roughly behaves like

$$
\frac{\omega_{n h}-\omega_{n}}{\omega_{n}} \approx C h^{\alpha}
$$

with a constant $C$ depending neither on the thickness $t$ nor on the mesh-size $h$ and an order of convergence $\alpha$ very close to 2 .

For each thickness $t$ and each vibration mode, we have estimated the order of convergence $\alpha$ and the constant $C$ by means of a least square fitting. We have also estimated by extrapolation the limit values for the Kirchhoff model $(t=0)$.

We summarize these results in Tables 8 10, separately for each vibration frequency. To allow for comparison, we report again normalized frequencies $\hat{\omega}_{n}:=$ $\omega_{n} / t$.

TABLE 8. Test 5: Normalized frequency $\hat{\omega}_{18}$ of simply supported laminated rectangular plates with varying thickness.

\begin{tabular}{lcccccc}
\hline Thickness & $N=16$ & $N=32$ & $N=64$ & Order & Exact & $C$ \\
\hline$t=0.1 \mathrm{~m}$ & 12224.615 & 11574.578 & 11416.650 & 1.88 & 11364.671 & 12.849 \\
$t=0.01 \mathrm{~m}$ & 12387.251 & 11714.984 & 11550.417 & 1.98 & 11496.221 & 13.347 \\
$t=0.001 \mathrm{~m}$ & 12388.971 & 11716.515 & 11551.887 & 1.98 & 11497.565 & 13.345 \\
$t=0.0001 \mathrm{~m}$ & 12388.988 & 11716.530 & 11551.896 & 1.98 & 11497.578 & 13.345 \\
\hline$t=0$ (extr.) & 12388.983 & 11716.534 & 11551.900 & 1.98 & 11497.579 & 13.345 \\
\hline
\end{tabular}

TABLE 9. Test 5: Normalized frequency $\hat{\omega}_{19}$ of simply supported laminated rectangular plates with varying thickness.

\begin{tabular}{lcccccc}
\hline Thickness & $N=16$ & $N=32$ & $N=64$ & Order & Exact & $C$ \\
\hline$t=0.1 \mathrm{~m}$ & 12234.055 & 11574.952 & 11416.664 & 1.99 & 11364.671 & 13.336 \\
$t=0.01 \mathrm{~m}$ & 12397.293 & 11715.159 & 11550.453 & 2.03 & 11496.221 & 13.543 \\
$t=0.001 \mathrm{~m}$ & 12399.027 & 11716.687 & 11551.926 & 2.03 & 11497.565 & 13.541 \\
$t=0.0001 \mathrm{~m}$ & 12399.044 & 11716.702 & 11551.940 & 2.03 & 11497.578 & 13.542 \\
\hline$t=0$ (extr.) & 12399.050 & 11716.706 & 11551.940 & 2.02 & 11497.579 & 13.541 \\
\hline
\end{tabular}

TABLE 10. Test 5: Normalized frequency $\hat{\omega}_{20}$ of simply supported laminated rectangular plates with varying thickness.

\begin{tabular}{lcccccc}
\hline Thickness & $N=16$ & $N=32$ & $N=64$ & Order & Exact & $C$ \\
\hline$t=0.1 \mathrm{~m}$ & 12239.736 & 11629.438 & 11477.448 & 2.04 & 11427.081 & 13.492 \\
$t=0.01 \mathrm{~m}$ & 12404.148 & 11771.789 & 11612.853 & 2.06 & 11560.081 & 13.620 \\
$t=0.001 \mathrm{~m}$ & 12405.903 & 11773.365 & 11614.372 & 2.05 & 11561.440 & 13.617 \\
$t=0.0001 \mathrm{~m}$ & 12405.921 & 11773.381 & 11614.385 & 2.05 & 11561.454 & 13.617 \\
\hline$t=0$ (extr.) & 12405.924 & 11773.377 & 11614.388 & 2.05 & 11561.454 & 13.617 \\
\hline
\end{tabular}

One conclusion clearly arises from these tables. The quality of the approximation does not deteriorate when computing frequencies of a cluster. In fact, the constants 
are essentially independent of $t$ and $h$ and the double order of convergence is preserved as the thickness becomes small (and consequently the vibration frequencies of the Reissner-Mindlin plate get closer).

\section{Conclusions}

We analyzed the problem of computing the vibration modes of a clamped laminated plate modeled by Reissner-Mindlin equations. We considered a finite-element method based on DL3 elements for the bending terms and standard triangular piecewise linear elements for the in-plane displacements. We proved optimal orders of convergence in $H^{1}$ and $L^{2}$ for displacements and rotations, as well as a double order for the eigenvalues. We also proved that the error estimates do not deteriorate as the thickness becomes small, which imply that the method is locking-free.

The keypoint of the proofs is an a priori estimate for the regularity of the solution of the corresponding load problem. This result is interesting beyond the present application, because the error of any numerical method for laminated plates relies on such an estimate

We reported numerical experiments confirming the theoretical results. Moreover, these experiments show the robustness with respect to the physical parameters, the type of meshes, and the interplay between membrane and bending behavior. The experiments also show that the method works for boundary conditions beyond the theoretical results.

In principle the analytical tools developed in this paper could be used to study the convergence of the method for more general boundary conditions and for laminated plates formed by anisotropic laminas, as well. However, a first step would be to provide a result analogous to that of the appendix, whose proof relies heavily on the Dirichlet character of the conditions and the isotropy of the laminas.

Finally, the experiments also show that the eigenvalue separation does not affect the accuracy of the method, although the constants in the error estimates in principle depend on the distance of the approximated eigenvalue to the rest of the spectrum. However, the performance of the method for clustered vibration frequencies is essentially the same as for isolated ones.

\section{ApPEndix}

In this Appendix we will obtain an a priori estimate for the solution of the load problem (2.2) similar to the one valid for classical homogeneous Reissner-Mindlin plates. With this purpose, first we prove the following auxiliary result.

Lemma 6.1. Let $\Omega$ be a convex polygonal domain in the plane. Given $F, G \in$ $L^{2}(\Omega)^{2}$, let $(u, \beta) \in H_{0}^{1}(\Omega)^{2} \times H_{0}^{1}(\Omega)^{2}$ be the unique solution of

$$
a((u, \beta),(v, \eta))=(F, v)+(G, \eta) \quad \forall(v, \eta) \in H_{0}^{1}(\Omega)^{2} \times H_{0}^{1}(\Omega)^{2} .
$$

Then, $(u, \beta) \in H^{2}(\Omega)^{2} \times H^{2}(\Omega)^{2}$ and

$$
\|u\|_{2}+\|\beta\|_{2} \leq C\left(\|F\|_{0}+\|G\|_{0}\right)
$$

with a constant $C$ independent of $F$ ad $G$.

Proof. We will resort to additional regularity results from [12, Section 8.6] regarding Dirichlet problems for elliptic systems. In this reference it is proved that the strip of the complex plane $|\operatorname{Re} \lambda| \leq 1$ is free of eigenvalues of the Mellin symbol, which 
implies $H^{2}$ regularity for $L^{2}$ right-hand sides, provided the elliptic system (6.1) is formally positive.

Let us recall that formally positiveness means in our case that if the bilinear form of the elliptic system is written as follows,

$$
a((u, \beta),(v, \eta))=\sum_{i, j=1}^{2} \boldsymbol{A}_{i j} \partial_{i} \boldsymbol{U} \partial_{j} \boldsymbol{V},
$$

with

$$
\boldsymbol{U}:=\left[\begin{array}{l}
u \\
\beta
\end{array}\right]: \Omega \longrightarrow \mathbb{R}^{4}, \quad \boldsymbol{V}:=\left[\begin{array}{l}
v \\
\eta
\end{array}\right]: \Omega \longrightarrow \mathbb{R}^{4},
$$

and $\boldsymbol{A}_{i j} \in \mathbb{R}^{4 \times 4}$, then

$$
\boldsymbol{A}:=\left[\begin{array}{ll}
\boldsymbol{A}_{11} & \boldsymbol{A}_{12} \\
\boldsymbol{A}_{21} & \boldsymbol{A}_{12}
\end{array}\right] \in \mathbb{R}^{8 \times 8}
$$

is a symmetric positive definite matrix.

In our case

$$
\begin{aligned}
a((u, \beta),(v, \eta))= & \bar{\lambda}_{1}(\operatorname{tr} \varepsilon(u), \operatorname{tr} \varepsilon(v))+2 \bar{\mu}_{1}(\varepsilon(u), \varepsilon(v)) \\
& +\bar{\lambda}_{2}(\operatorname{tr} \varepsilon(u), \operatorname{tr} \varepsilon(\eta))+2 \bar{\mu}_{2}(\varepsilon(u), \varepsilon(\eta)) \\
& +\bar{\lambda}_{2}(\operatorname{tr} \varepsilon(v), \operatorname{tr} \varepsilon(\beta))+2 \bar{\mu}_{2}(\varepsilon(v), \varepsilon(\beta)) \\
& +\bar{\lambda}_{3}(\operatorname{tr} \varepsilon(\beta), \operatorname{tr} \varepsilon(\eta))+2 \bar{\mu}_{3}(\varepsilon(\beta), \varepsilon(\eta)),
\end{aligned}
$$

with

$$
\begin{array}{lll}
\bar{\lambda}_{1}=\frac{\lambda_{1}+\lambda_{2}}{2}, & \bar{\lambda}_{2}=\frac{\lambda_{1}-\lambda_{2}}{8}, & \bar{\lambda}_{3}=\frac{\lambda_{1}+\lambda_{2}}{24}, \\
\bar{\mu}_{1}=\frac{\mu_{1}+\mu_{2}}{2}, & \bar{\mu}_{2}=\frac{\mu_{1}-\mu_{2}}{8}, & \bar{\mu}_{3}=\frac{\mu_{1}+\mu_{2}}{24} .
\end{array}
$$

If (6.3) is directly written in the form (6.2), the resulting matrix $\boldsymbol{A}$ is not positive definite. However, using the fact that

$$
\begin{aligned}
& \int_{\Omega} \partial_{2} u_{1} \partial_{1} \eta_{2}=\int_{\Omega} \partial_{1} u_{1} \partial_{2} \eta_{2}, \quad \quad \int_{\Omega} \partial_{1} u_{2} \partial_{2} \eta_{1}=\int_{\Omega} \partial_{2} u_{2} \partial_{1} \eta_{1}, \\
& \int_{\Omega} \partial_{2} \beta_{1} \partial_{1} v_{2}=\int_{\Omega} \partial_{1} \beta_{1} \partial_{2} v_{2}, \quad \text { and } \quad \int_{\Omega} \partial_{1} \beta_{2} \partial_{2} v_{1}=\int_{\Omega} \partial_{2} \beta_{2} \partial_{1} v_{1}
\end{aligned}
$$

(which is proved by a double integration by parts), (6.3) can also be written in the form (6.2) with

$$
\boldsymbol{A}_{11}=\left[\begin{array}{cccc}
\bar{\lambda}_{1}+2 \bar{\mu}_{1} & 0 & \bar{\lambda}_{2}+2 \bar{\mu}_{2} & 0 \\
0 & \bar{\mu}_{1} & 0 & \bar{\mu}_{2} \\
\bar{\lambda}_{2}+2 \bar{\mu}_{2} & 0 & \bar{\lambda}_{3}+2 \bar{\mu}_{3} & 0 \\
0 & \bar{\mu}_{2} & 0 & \bar{\mu}_{3}
\end{array}\right], \quad \boldsymbol{A}_{22}=\left[\begin{array}{cccc}
\bar{\mu}_{1} & 0 & \bar{\mu}_{2} & 0 \\
0 & \bar{\lambda}_{1}+2 \bar{\mu}_{1} & 0 & \bar{\lambda}_{2}+2 \bar{\mu}_{2} \\
\bar{\mu}_{2} & 0 & \bar{\mu}_{3} & 0 \\
0 & \bar{\lambda}_{2}+2 \bar{\mu}_{2} & 0 & \bar{\lambda}_{3}+2 \bar{\mu}_{3}
\end{array}\right],
$$

and

$$
\boldsymbol{A}_{12}=\boldsymbol{A}_{21}^{\mathrm{t}}=\left[\begin{array}{cccc}
0 & \bar{\lambda}_{1}+\bar{\mu}_{1} & 0 & \bar{\lambda}_{2}+\bar{\mu}_{2} \\
0 & 0 & 0 & 0 \\
0 & \bar{\lambda}_{2}+\bar{\mu}_{2} & 0 & \bar{\lambda}_{3}+\bar{\mu}_{3} \\
0 & 0 & 0 & 0
\end{array}\right]
$$


There only remains to prove that $\boldsymbol{A}$ is positive definite. With this purpose, we write $\boldsymbol{A}=\boldsymbol{B}_{1}+\boldsymbol{B}_{2}$ with

$$
\boldsymbol{B}_{1}:=\left[\begin{array}{cccccccc}
2 \bar{\mu}_{1} & 0 & 2 \bar{\mu}_{2} & 0 & 0 & \bar{\mu}_{1} & 0 & \bar{\mu}_{2} \\
0 & \bar{\mu}_{1} & 0 & \bar{\mu}_{2} & 0 & 0 & 0 & 0 \\
2 \bar{\mu}_{2} & 0 & 2 \bar{\mu}_{3} & 0 & 0 & \bar{\mu}_{2} & 0 & \bar{\mu}_{3} \\
0 & \bar{\mu}_{2} & 0 & \bar{\mu}_{3} & 0 & 0 & 0 & 0 \\
0 & 0 & 0 & 0 & \bar{\mu}_{1} & 0 & \bar{\mu}_{2} & 0 \\
\bar{\mu}_{1} & 0 & \bar{\mu}_{2} & 0 & 0 & 2 \bar{\mu}_{1} & 0 & 2 \bar{\mu}_{2} \\
0 & 0 & 0 & 0 & \bar{\mu}_{2} & 0 & \bar{\mu}_{3} & 0 \\
\bar{\mu}_{2} & 0 & \bar{\mu}_{3} & 0 & 0 & 2 \bar{\mu}_{2} & 0 & 2 \bar{\mu}_{3}
\end{array}\right]
$$

and

$$
\boldsymbol{B}_{2}:=\left[\begin{array}{cccccccc}
\bar{\lambda}_{1} & 0 & \bar{\lambda}_{2} & 0 & 0 & \bar{\lambda}_{1} & 0 & \bar{\lambda}_{2} \\
0 & 0 & 0 & 0 & 0 & 0 & 0 & 0 \\
\bar{\lambda}_{2} & 0 & \bar{\lambda}_{3} & 0 & 0 & \bar{\lambda}_{2} & 0 & \bar{\lambda}_{3} \\
0 & 0 & 0 & 0 & 0 & 0 & 0 & 0 \\
0 & 0 & 0 & 0 & 0 & 0 & 0 & 0 \\
\bar{\lambda}_{1} & 0 & \bar{\lambda}_{2} & 0 & 0 & \bar{\lambda}_{1} & 0 & \bar{\lambda}_{2} \\
0 & 0 & 0 & 0 & 0 & 0 & 0 & 0 \\
\bar{\lambda}_{2} & 0 & \bar{\lambda}_{3} & 0 & 0 & \bar{\lambda}_{2} & 0 & \bar{\lambda}_{3}
\end{array}\right]
$$

We observe that $\boldsymbol{B}_{1}$ is positive definite. In fact, reordering rows and columns of $\boldsymbol{B}_{1}$, we obtain the block diagonal matrix

$$
\widehat{B}_{1}:=\left[\begin{array}{cc}
\boldsymbol{C}_{1} & \boldsymbol{O} \\
\boldsymbol{O} & \boldsymbol{C}_{2}
\end{array}\right]
$$

with

$$
\boldsymbol{C}_{1}:=\left[\begin{array}{cc}
2 \boldsymbol{D}_{1} & \boldsymbol{D}_{1} \\
\boldsymbol{D}_{1} & 2 \boldsymbol{D}_{1}
\end{array}\right], \quad \boldsymbol{C}_{2}:=\left[\begin{array}{cc}
\boldsymbol{D}_{1} & \boldsymbol{O} \\
\boldsymbol{O} & \boldsymbol{D}_{1}
\end{array}\right], \quad \text { and } \quad \boldsymbol{D}_{1}:=\left[\begin{array}{cc}
\bar{\mu}_{1} & \bar{\mu}_{2} \\
\bar{\mu}_{2} & \bar{\mu}_{3}
\end{array}\right] .
$$

Using (6.4), it is simple to show that $\bar{\mu}_{1} \bar{\mu}_{3}-\bar{\mu}_{2}^{2}>0$. Hence, $\boldsymbol{D}_{1}$ is positive definite. Therefore, elementary computations show that $\boldsymbol{C}_{1}, \boldsymbol{C}_{2}$, and consequently $\widehat{\boldsymbol{B}}_{1}$ and $\boldsymbol{B}_{1}$, are positive definite, too.

On the other hand, $\boldsymbol{B}_{2}$ is positive semi-definite. In fact, reordering rows and columns of $\boldsymbol{B}_{2}$, we obtain

$$
\widehat{\boldsymbol{B}}_{2}=\left[\begin{array}{ll}
\boldsymbol{C}_{3} & \boldsymbol{O} \\
\boldsymbol{O} & \boldsymbol{O}
\end{array}\right], \quad \text { with } \quad \boldsymbol{C}_{3}:=\left[\begin{array}{ll}
\boldsymbol{D}_{2} & \boldsymbol{D}_{2} \\
\boldsymbol{D}_{2} & \boldsymbol{D}_{2}
\end{array}\right] \quad \text { and } \quad \boldsymbol{D}_{2}:=\left[\begin{array}{ll}
\bar{\lambda}_{1} & \bar{\lambda}_{2} \\
\bar{\lambda}_{2} & \bar{\lambda}_{3}
\end{array}\right] .
$$

The matrix $\boldsymbol{D}_{2}$ is positive definite because, using again (6.4), we have $\bar{\lambda}_{1} \bar{\lambda}_{3}-\bar{\lambda}_{2}^{2}>0$. Therefore, $\boldsymbol{C}_{3}$ and consequently $\widehat{\boldsymbol{B}}_{2}$ and $\boldsymbol{B}_{2}$ are positive semi-definite, too. Thus, $\boldsymbol{A}=\boldsymbol{B}_{1}+\boldsymbol{B}_{2}$ is positive definite and we conclude the proof.

Now we are in a position to prove that the solutions of the load problems for laminated and classical homogeneous Reissner-Mindlin clamped plates have the same regularity. With this aim, we adapt the arguments from the proof of [1, Theorem 7.1].

For any $t>0$ and $(f, m, g) \in H$, let $(u, \beta, w) \in V$ and $\gamma \in L^{2}(\Omega)$ be the solution to

$$
\left\{\begin{array}{l}
a((u, \beta),(v, \eta))+(\gamma, \eta-\nabla z)=(f, v)+(m, \eta)+(g, z) \quad \forall(v, \eta, z) \in V \\
\gamma=\frac{\kappa}{t^{2}}(\beta-\nabla w)
\end{array}\right.
$$


Consider the Helmholtz decomposition of the shear term $\gamma \in H_{0}(\operatorname{rot}, \Omega)$ :

$$
\gamma=\nabla r+\operatorname{curl} p
$$

with $r \in H_{0}^{1}(\Omega)$ and $p \in H^{1}(\Omega) / \mathbb{R}$. Using this decomposition in (6.5), this problem turns out equivalent to finding $r, w \in H_{0}^{1}(\Omega), u, \beta \in H_{0}^{1}(\Omega)^{2}$, and $p \in H^{1}(\Omega) / \mathbb{R}$ such that

$$
\begin{aligned}
& -(\nabla r, \nabla z)=(g, z) \quad \forall z \in H_{0}^{1}(\Omega), \\
& a((u, \beta),(v, \eta))+(\operatorname{curl} p, \eta)=(f, v)+(m, \eta)-(\nabla r, \eta) \\
& \forall(v, \eta) \in H_{0}^{1}(\Omega)^{2} \times H_{0}^{1}(\Omega)^{2}, \\
& (\beta, \operatorname{curl} q)-\frac{t^{2}}{\kappa}(\operatorname{curl} p, \operatorname{curl} q)=0 \quad \forall q \in H^{1}(\Omega) / \mathbb{R}, \\
& (\nabla w, \nabla s)=(\beta, \nabla s)-\frac{t^{2}}{\kappa}(\nabla r, \nabla s) \quad \forall s \in H_{0}^{1}(\Omega) .
\end{aligned}
$$

This is a well-posed problem. In fact, first, $r$ is the unique solution to the Dirichlet problem (6.7). Then, subtracting (6.9) from (6.8), since $t>0$, existence and uniqueness of $(u, \beta)$ and $p$ follows from Lax-Milgram lemma. Finally, $w$ is the solution to the well-posed problem (6.10).

For $t=0$, equations (6.7)-(6.10) also make sense, but the terms $(\operatorname{curl} p, \eta)$ from (6.8) and $(\beta, \operatorname{curl} q)$ from (6.9) must be understood weakly. Thus, in this case, we are led to finding $r_{0}, w_{0} \in H_{0}^{1}(\Omega), u_{0}, \beta_{0} \in H_{0}^{1}(\Omega)^{2}$, and $p_{0} \in L^{2}(\Omega) / \mathbb{R}$ such that

$$
\begin{aligned}
& \text { - }\left(\nabla r_{0}, \nabla z\right)=(g, z) \quad \forall z \in H_{0}^{1}(\Omega), \\
& a\left(\left(u_{0}, \beta_{0}\right),(v, \eta)\right)+\left(p_{0}, \operatorname{rot} \eta\right)=(f, v)+(m, \eta)-\left(\nabla r_{0}, \eta\right) \\
& \forall(v, \eta) \in H_{0}^{1}(\Omega)^{2} \times H_{0}^{1}(\Omega)^{2}, \\
& \left(\operatorname{rot} \beta_{0}, q\right)=0 \quad \forall q \in L^{2}(\Omega) / \mathbb{R} . \\
& \left(\nabla w_{0}, \nabla s\right)=\left(\beta_{0}, \nabla s\right) \quad \forall s \in H_{0}^{1}(\Omega) \text {. }
\end{aligned}
$$

This is a well-posed problem, too. In fact, equations (6.11) and (6.7) are the same, so that $r_{0}=r$, as well. Existence and uniqueness of $\left(u_{0}, \beta_{0}\right)$ and $p_{0}$ follow from the fact that (6.12) - (6.13) is a well-posed mixed problem ( $a$ is elliptic in the whole space and the inf-sup condition is essentially the same as that of the Stokes problem). Finally, $w_{0}$ is the solution of the Dirichlet problem (6.14). Moreover, the following additional regularity holds.

Lemma 6.2. Let $\left(r_{0}, u_{0}, \beta_{0}, p_{0}, w_{0}\right)$ be the solution to problem (6.11) (6.14). Then, $r_{0}, w_{0} \in H^{2}(\Omega), u_{0}, \beta_{0} \in H^{2}(\Omega)^{2}, p_{0} \in H^{1}(\Omega)$, and

$$
\left\|r_{0}\right\|_{2}+\left\|u_{0}\right\|_{2}+\left\|\beta_{0}\right\|_{2}+\left\|p_{0}\right\|_{1}+\left\|w_{0}\right\|_{2} \leq C\|(f, m, g)\|_{0},
$$

with a constant $C$ independent of $f, m$, and $g$.

Proof. The standard a priori estimate for the Poisson equation on a convex domain, implies that $r_{0} \in H^{2}(\Omega)$ and

$$
\left\|r_{0}\right\|_{2} \leq C\|g\|_{0}
$$


Next, we write (6.12) -6.13) in the distributional sense:

$$
\left\{\begin{array}{lr}
-\bar{\mu}_{1} \Delta u_{0}-\left(\bar{\lambda}_{1}+\bar{\mu}_{1}\right) \nabla\left(\operatorname{div} u_{0}\right)-\bar{\mu}_{2} \Delta \beta_{0}-\left(\bar{\lambda}_{2}+\bar{\mu}_{2}\right) \nabla\left(\operatorname{div} \beta_{0}\right)=f, \\
-\bar{\mu}_{2} \Delta u_{0}-\left(\bar{\lambda}_{2}+\bar{\mu}_{2}\right) \nabla\left(\operatorname{div} u_{0}\right)-\bar{\mu}_{3} \Delta \beta_{0}-\left(\bar{\lambda}_{3}+\bar{\mu}_{3}\right) \nabla\left(\operatorname{div} \beta_{0}\right) \\
\operatorname{courl} p_{0}=m-\nabla r_{0},
\end{array}\right.
$$

with Dirichlet homogeneous boundary conditions for $u_{0}$ and $\beta_{0}$ and the coefficients $\bar{\lambda}_{i}$ and $\bar{\mu}_{i}, i=1,2,3$, as defined in (6.4).

The third equation above implies that $\beta_{0}=\nabla \varphi$, with $\varphi \in H_{0}^{1}(\Omega)$ such that $\frac{\partial \varphi}{\partial n}=0$ on $\partial \Omega$. Using this in the first and second equations, we obtain

$$
\begin{array}{r}
-\bar{\mu}_{1} \Delta u_{0}-\left(\bar{\lambda}_{1}+\bar{\mu}_{1}\right) \nabla\left(\operatorname{div} u_{0}\right)-\left(\bar{\lambda}_{2}+2 \bar{\mu}_{2}\right) \Delta(\nabla \varphi)=f, \\
-\bar{\mu}_{2} \Delta u_{0}-\left(\bar{\lambda}_{2}+\bar{\mu}_{2}\right) \nabla\left(\operatorname{div} u_{0}\right)-\left(\bar{\lambda}_{3}+2 \bar{\mu}_{3}\right) \Delta(\nabla \varphi) \\
+\operatorname{curl} p_{0}=m-\nabla r_{0},
\end{array}
$$

with homogeneous boundary conditions $u_{0}=0, \beta_{0}=0, \varphi=0$ and $\frac{\partial \varphi}{\partial n}=0$ on $\partial \Omega$.

Taking divergence in the first two equations, we have

$$
\begin{aligned}
& -\left(\bar{\lambda}_{1}+2 \bar{\mu}_{1}\right) \operatorname{div}\left(\Delta u_{0}\right)-\left(\bar{\lambda}_{2}+2 \bar{\mu}_{2}\right) \Delta^{2} \varphi=\operatorname{div} f, \\
& -\left(\bar{\lambda}_{2}+2 \bar{\mu}_{2}\right) \operatorname{div}\left(\Delta u_{0}\right)-\left(\bar{\lambda}_{3}+2 \bar{\mu}_{3}\right) \Delta^{2} \varphi=\operatorname{div} m-\Delta r_{0} .
\end{aligned}
$$

Eliminating $u_{0}$ we arrive at the following problem for $\varphi$ :

$$
\left\{\begin{array}{l}
K \Delta^{2} \varphi=\left(\bar{\lambda}_{2}+2 \bar{\mu}_{2}\right) \operatorname{div} f-\left(\bar{\lambda}_{1}+2 \bar{\mu}_{1}\right)\left(\operatorname{div} m-\Delta r_{0}\right) \in H^{-1}(\Omega), \\
\varphi=\frac{\partial \varphi}{\partial n}=0 \quad \text { on } \partial \Omega .
\end{array}\right.
$$

with $K:=\left(2 \bar{\mu}_{1}+\bar{\lambda}_{1}\right)\left(2 \bar{\mu}_{3}+\bar{\lambda}_{3}\right)-\left(2 \bar{\mu}_{2}+\bar{\lambda}_{2}\right)^{2}$, which can be shown to be strictly positive by using (6.4) and a little algebra. Therefore, from the standard a priori estimate for the biharmonic equation in convex domains, we know that $\varphi \in H^{3}(\Omega)$ and

$$
\|\varphi\|_{3} \leq C\left(\|\operatorname{div} f\|_{-1}+\|\operatorname{div} m\|_{-1}+\left\|\Delta r_{0}\right\|_{0}\right) \leq C\|(f, m, g)\|_{0},
$$

where we have used (6.16) for the last inequality. Therefore $\beta_{0}=\nabla \varphi \in H^{2}(\Omega)^{2}$.

Next, using the last inequality in (6.17), we obtain from the usual a priori estimate for the elasticity problem in a polygonal convex domain that $u_{0} \in H^{2}(\Omega)^{2}$ and

$$
\left\|u_{0}\right\|_{2} \leq C\|(f, m, g)\|_{0} .
$$

Now, from this inequality and (6.18), we obtain that $p_{0} \in H^{1}(\Omega)$ and the corresponding estimate. Finally, the regularity of $w_{0}$ follows again from the standard a priori estimate for the Poisson equation on a convex domain applied to (6.14). Thus we conclude the proof.

Corollary 6.3. The solution of the limit problem (2.4) satisfies $u_{0}, \beta_{0} \in H^{2}(\Omega)^{2}$, $w_{0} \in H^{2}(\Omega), \gamma_{0} \in L^{2}(\Omega)^{2}$, and the a priori estimate (2.5) holds true.

Proof. In this case, the Helmholtz decomposition (6.6) holds in a distributional sense (cf. 6, Proposition 3.4]):

$$
\gamma_{0}=\nabla r_{0}+\operatorname{curl} p_{0}
$$

with $r_{0} \in H_{0}^{1}(\Omega)$ and $p_{0} \in L^{2}(\Omega) / \mathbb{R}$. Then, problem (2.4) is equivalent to (6.11)(6.14), with $f=m=0$. Hence, the additional regularity result follows from Lemma 6.2 and the above equation. 
Now we are ready to prove the a priori estimate for the solution to problem (6.7)(6.10) and, consequently, to that of problem (6.5), which is the main goal of this Appendix.

Lemma 6.4. For any $t>0$, let $(r, u, \beta, p, w)$ be the solution to problem (6.7)(6.10). Then, $r, w, p \in H^{2}(\Omega), u, \beta \in H^{2}(\Omega)^{2}$, and

$$
\|r\|_{2}+\|u\|_{2}+\|\beta\|_{2}+\|p\|_{1}+t\|p\|_{2}+\|w\|_{2} \leq C\left(\|f\|_{0}+\|m\|_{0}+\|g\|_{0}\right),
$$

with a constant $C$ independent of $t, f, m$, and $g$.

Proof. Let $\left(r_{0}, u_{0}, \beta_{0}, p_{0}, w_{0}\right)$ be the solution to problem (6.11)-(6.14). Recall that $r=r_{0}$, so that we have already proved in Lemma 6.2 the estimate for $r$ (cf. (6.16)).

Now, since according to Lemma 6.2. $p_{0} \in H^{1}(\Omega)$, from (6.8)-(6.9) and (6.12) (6.13), we obtain

$$
\begin{aligned}
a\left(\left(u-u_{0}, \beta-\beta_{0}\right),(v, \eta)\right) & +\left(\operatorname{curl}\left(p-p_{0}\right), \eta\right)-\left(\beta-\beta_{0}, \operatorname{curl} q\right) \\
& +\frac{t^{2}}{\kappa}\left(\operatorname{curl}\left(p-p_{0}\right), \operatorname{curl} q\right)=-\frac{t^{2}}{\kappa}\left(\operatorname{curl} p_{0}, \operatorname{curl} q\right)
\end{aligned}
$$

for all $v, \eta \in H_{0}^{1}(\Omega)^{2}$ and all $q \in H^{1}(\Omega) / \mathbb{R}$. Testing this equation with $v:=u-u_{0}$, $\eta:=\beta-\beta_{0}$, and $q=: p-p_{0}$ and using the ellipticity of $a$, we have

$$
\left\|u-u_{0}\right\|_{1}^{2}+\left\|\beta-\beta_{0}\right\|_{1}^{2}+t^{2}\left\|p-p_{0}\right\|_{1}^{2} \leq C t^{2}\left\|p_{0}\right\|_{1}\left\|p-p_{0}\right\|_{1}
$$

and, from this estimate and (6.15), we arrive at

$$
\left\|u-u_{0}\right\|_{1}+\left\|\beta-\beta_{0}\right\|_{1}+t\left\|p-p_{0}\right\|_{1} \leq C t\|(f, m, g)\|_{0} .
$$

Hence, also,

$$
\|p\|_{1} \leq\left\|p-p_{0}\right\|_{1}+\left\|p_{0}\right\|_{1} \leq C\|(f, m, g)\|_{0} .
$$

Next, we apply Lemma 6.1 to (6.8) with $F:=f$ and $G:=m-\nabla r-\operatorname{curl} p$, to show that $u, \beta \in H^{2}(\Omega)^{2}$ and

$$
\|u\|_{2}+\|\beta\|_{2} \leq C\|(f, m, g)\|_{0}
$$

where we have used (6.16) and (6.20).

On the other hand, from (6.9) and (6.13), we have that

$$
(\operatorname{curl} p, \operatorname{curl} q)=\frac{\kappa}{t^{2}}\left(\beta-\beta_{0}, \operatorname{curl} q\right)=\left(\operatorname{rot}\left(\beta-\beta_{0}\right), q\right) \quad \forall q \in H^{1}(\Omega) .
$$

Therefore, $p$ is the solution of the Neumann problem

$$
\left\{\begin{array}{l}
\Delta p=\frac{\kappa}{t^{2}} \operatorname{rot}\left(\beta-\beta_{0}\right) \quad \text { in } \Omega \\
\frac{\partial p}{\partial n}=0 \quad \text { on } \partial \Omega
\end{array}\right.
$$

Hence, since $\Omega$ is convex we have

$$
\|p\|_{2} \leq C t^{-2}\left\|\beta-\beta_{0}\right\|_{1} \leq C t^{-1}\|(f, m, g)\|_{0},
$$

the last inequality because of (6.19).

Finally, (6.10) is a Poisson equation, for which there holds the a priori estimate

$$
\|w\|_{2} \leq C\left(\|\beta\|_{1}+t^{2}\|r\|_{2}\right) \leq C\|(f, m, g)\|_{0},
$$

where we have used (6.16) once more. Thus we end the proof.

We conclude with the main result of this Appendix. 
Theorem 6.5. Let $\Omega$ be a convex polygonal domain in the plane. For any $t>0$ and $(f, m, g) \in H$, let $(u, \beta, w) \in V$ and $\gamma \in L^{2}(\Omega)$ be the solution to problem (6.5). Then, $u, \beta \in H^{2}(\Omega)^{2}, w \in H^{2}(\Omega), \gamma \in H^{1}(\Omega)^{2}$, and there exists a constant $C$, independent of $t$ and $(f, m, g)$, such that

$$
\|u\|_{2}+\|\beta\|_{2}+\|w\|_{2}+\|\gamma\|_{0}+t\|\gamma\|_{1} \leq C\left(\|f\|_{0}+\|m\|_{0}+\|g\|_{0}\right) .
$$

Proof. This is an immediate consequence of Lemma 6.4 and the equivalence between problems (6.5) and (6.7)- (6.10), through (6.6).

Corollary 6.6. The solution of the load problem (2.2) satisfies $u, \beta \in H^{2}(\Omega)^{2}$, $w \in H^{2}(\Omega), \gamma \in H^{1}(\Omega)^{2}$, and the a priori estimate (2.3) holds true.

Proof. It is a direct application of the previous theorem to the particular right-hand side of the first equation from (2.2).

\section{ACKNOWLEDGMENT}

The authors thank Monique Dauge for suggesting the use of reference 12 to prove the regularity results in the Appendix and Carlos Reales for providing the axisymmetric 3D code for the numerical test from Section 4.3 .

\section{REFERENCES}

[1] D.N. Arnold and R.S. Falk, A uniformly accurate finite element method for the ReissnerMindlin plate, SIAM J. Numer. Anal., 26 (1989) 1276-1290. MR.1025088 (91c:65068)

[2] F. Auricchio, C. Lovadina, and E. Sacco, Analysis of mixed finite elements for laminated composite plates, Comput. Methods Appl. Mech. Engrg., 190 (2000) 4767-4783. MR.1840799 (2002d:74062)

[3] F. Auricchio and E. Sacco, A mixed-enhanced finite element for the analysis of laminated composite plates, Internat. J. Numer. Methods Engrg., 44 (1999) 1481-1504.

[4] I. Babuška and J. Osborn, Eigenvalue problems, in Handbook of Numerical Analysis, Vol. II, P.G. Ciarlet and J.L. Lions, eds., North-Holland, Amsterdam, 1991, pp. 641-787. MR 1115240

[5] K.J. Bathe and E.N. Dvorkin, A four-node plate bending element based on Mindlin/Reissner plate theory and a mixed interpolation, Internat. J. Numer. Methods Engrg., 21 (1985) 367383.

[6] F. Brezzi and M. Fortin, Mixed and Hybrid Finite Element Methods, Springer-Verlag, New York, 1991. MR1115205(92d:65187)

[7] D. Chenais and J.-C. Paumier, On the locking phenomenon for a class of elliptic problems, Numer. Math., 67 (1994) 427-440. MR1274440 (95g:65147)

[8] R. Durán, E. Hernández, L. Hervella-Nieto, E. Liberman, and R. Rodríguez, Error estimates for low-order isoparametric quadrilateral finite elements for plates, SIAM J. Numer. Anal., 41 (2003) 1751-1772. MR2035005 (2004m:65192)

[9] R. Durán, L. Hervella-Nieto, E. Liberman, R. Rodríguez, and J. Solomin, Approximation of the vibration modes of a plate by Reissner-Mindlin equations, Math. Comp., 68 (1999) 1447-1463. MR:1648387 (99m:73045)

[10] R. Durán and E. Liberman, On mixed finite element methods for Reissner Mindlin Plate model, Math. Comp., 58 (1992) 561-573. MR.1106965 (92f:65135)

[11] T. Kato, Perturbation Theory for Linear Operators, Springer-Verlag, Berlin, 1995. MR.1335452 (96a:47025)

[12] V. Kozlov, V. Maz'ya, and J. Rossmann, Spectral Problems Associated with Corner Singularities of Solutions to Elliptic Equations, Mathematical Surveys and Monographs 85, AMS, Providence, RI, 2001. MR1788991 (2001i:35069)

[13] O.O. Ochoa and J.N. Reddy, Finite element analysis of composite laminates, Kluwer Academic Publishers, Dordrecht, The Netherlands, 1992.

[14] P.A. Raviart and J.M. Thomas, A mixed finite element method for 2nd order elliptic problems, in Mathematical Aspects of Finite Element Methods, Lecture Notes in Mathematics 606, Springer, Berlin, 1977, pp. 292-315. MR0483555 (58:3547) 
[15] J.N. Reddy, Energy and Variational Methods in Applied Mechanics, Wiley, New York, 1984.

[16] J.N. Reddy, Mechanics of Laminated Composite Plates - Theory and Analysis, CRC Press, Boca Raton, 1997.

Departamento de Matemática, Facultad de Ciencias Exactas y Naturales, UniversiDad de Buenos Aires, 1428, Buenos Aires, Argentina

E-mail address: rduran@dm.uba.ar

CI $^{2}$ Ma, Departamento de Ingeniería Matemática, Universidad de Concepción, Casilla 160-C, Concepción, Chile.

E-mail address: rodolfo@ing-mat.udec.cl

Escuela de Obras Civiles, Universidad Andres Bello, Autopista 7100, Concepción, CHILE.

E-mail address: fsanhueza@unab.cl 